\title{
CoBaltDB: Complete bacterial and archaeal orfeomes subcellular localization database and associated resources
}

\author{
David Goudenège, Stéphane Avner, Céline Lucchetti-Miganeh, Frédérique Barloy-Hubler
}

\begin{abstract}
Background: The functions of proteins are strongly related to their localization in cell compartments (for example the cytoplasm or membranes) but the experimental determination of the sub-cellular localization of proteomes is laborious and expensive. A fast and low-cost alternative approach is in silico prediction, based on features of the protein primary sequences. However, biologists are confronted with a very large number of computational tools that use different methods that address various localization features with diverse specificities and sensitivities. As a result, exploiting these computer resources to predict protein localization accurately involves querying all tools and comparing every prediction output; this is a painstaking task. Therefore, we developed a comprehensive database, called CoBaltDB, that gathers all prediction outputs concerning complete prokaryotic proteomes.

Description: The current version of CoBaltDB integrates the results of 43 localization predictors for 784 complete bacterial and archaeal proteomes (2.548.292 proteins in total). CoBaltDB supplies a simple user-friendly interface for retrieving and exploring relevant information about predicted features (such as signal peptide cleavage sites and transmembrane segments). Data are organized into three work-sets ("specialized tools", "meta-tools" and "additional tools"). The database can be queried using the organism name, a locus tag or a list of locus tags and may be browsed using numerous graphical and text displays.

Conclusions: With its new functionalities, CoBaltDB is a novel powerful platform that provides easy access to the results of multiple localization tools and support for predicting prokaryotic protein localizations with higher confidence than previously possible. CoBaltDB is available at http://www.umr6026.univ-rennes1.fr/english/home/ research/basic/software/cobalten.
\end{abstract}

\section{Background}

Determining the subcellular localization of proteins is essential for the functional annotation of proteomes $[1,2]$. Bacterial proteins can exist in soluble (i.e free) forms in cellular spaces (cytoplasm in both monoderm and diderm bacteria and periplasm in diderms), anchored to membranes (cytoplasm membrane in monoderms, inner- or outer membrane in diderms) or cell wall (in monoderms). They can also be released into the extracellular environment or directly translocated into host cells [3]. All protein synthesis takes place in the cytoplasm, so all non-cytoplasmic proteins must pass through one or two lipid bilayers by a mechanism

\footnotetext{
* Correspondence: fhubler@univ-rennes1.fr

CNRS UMR 6026, ICM, Equipe B@SIC, Université de Rennes 1, Campus de Beaulieu, 35042 Rennes, France
}

commonly called "secretion". Protein secretion is involved in various processes including plant-microbe interactions [4,5]), biofilm formation [6,7] and virulence of plant and human pathogens [8-10]. Two main systems are involved in protein translocation across the cytoplasmic membrane, namely the essential and universal Sec (Secretion) pathway and the Tat (Twin-arginine translocation) pathway found in some prokaryotes (monoderms and diderms) and eukaryotes alike [11-16]. The Sec machinery recognizes an N-terminal hydrophobic signal sequence and translocates unfolded proteins [12], whereas the Tat machinery recognizes a basic-rich $\mathrm{N}$-terminal motif (SRR-x-FLK) and transports fully folded proteins $[13,14])$. In addition to these systems, diderm bacteria have six further systems that secrete proteins using a contiguous channel spanning the two

\section{() Biomed Central}


membranes (T1SS, $[17,18]$, T3SS, T4SS and T6SS [19-24]) or in two steps, the first being Sec- or Tatdependent export into the periplasmic and the second being translocation across the outer membrane (T2SS, [25-27] and T5SS, [28,29]). Other diderm protein secretion systems exist: they include the chaperone-usher system (CU or T7SS, $[30,31]$ ) and the extracellular nucleation-precipitation mechanism (ENP or T8SS, [32]). It is worth mentioning that the terminology T7SS has also been proposed to describe a completely different protein secretion system, namely the ESAT-6 protein secretion (ESX) in Mycobacteria, now considered as diderm bacteria [33]. Beside Sec and Tat pathways, monoderm bacteria have additional secretion systems for protein translocation across the cytoplasmic membrane, namely the flagella export apparatus (FEA [34]), the fimbrilin-protein exporter (FPE, [35,36]) and the WXG100 secretion system (Wss, [37,38]).

Establishing whole proteome subcellular localization by biochemical experiments is possible but arduous, time consuming and expensive. Data concerning predicted proteins (from whole genome sequences) is continuously increasing. High-throughput in silico analysis is required for fast and accurate prediction of additional attributes based solely on their amino acid sequences. There are large numbers of global (that yield final localization) and specialized (that predict features) tools for computer-assisted prediction of protein localizations. Most specialized tools tend to detect the presence of $\mathrm{N}$ terminal signal peptides (SP). Prediction of Sec-sorting signals has a long history as the first methods, based on weight matrices, were published about fifteen years ago [39-41]. Numerous machine learning-based methods are now available [42-50]. The distinction between Tat- and Sec- sorting signals is essentially based on the recognition, in the $n / h$ regions edge, of the twin-arginine motif [51], using regular expressions combined with hydrophobicity measures [52] or machine learning [53]. Prelipoproteins SP have the same $\mathrm{n}$ - and $\mathrm{h}$ - regions as Sec SP but contain, in the c-region, a well-conserved lipobox [54], recognized for cleavage by the type II signal peptidase [55]. Lipoprotein prediction tools use regular expression patterns to detect this lipobox [56,57], combined with Hidden Markov Models (HMM) [58] or Neural Networks (NN) [59]. Other attributes predicted by specialized tools are $\alpha$-helices and $\beta$-barrel transmembrane segments. In 1982, Kyte and Doolittle proposed a hydropathy-based method to predict transmembrane (TM) helices in a protein sequence. This approach was enhanced by combining discriminant analysis [60], hydrophobicity scales [61-63] amino acid properties $[64,65]$. Complex algorithms are also available and employ statistics [66], multiple sequence alignments [67] and machine learning approaches [68-73]. $\beta$-barrel segments, embedded in outer membrane proteins, are harder to predict than $\alpha$-helical segments, mostly because they are shorter; nevertheless, many methods are available based on similar strategies [74-87].

This plethora of protein localization predictors and databases [88-91] constitutes an important resource but requires time and expertise for efficient exploitation. Some of the tools require computing skills, as they have to be locally installed; others are difficult to use (numerous parameters) or to interpret (large quantities of graphics and output data). Web tools are disseminated and need numerous manual requests. Additionally, researchers have to decide which of these numerous tools are the most pertinent for their purposes, and selection is problematic without appropriate training sets. Recent work shows that the best strategy for exploiting the various tools is to compare them [92-94].

Here, we describe CoBaltDB, the first public database that displays the results obtained by 43 localization predictor tools for 776 complete prokaryotic proteomes. CoBaltDB will help microbiologists explore and analyze subcellular localization predictions for all proteins predicted from a complete genome; it should thereby facilitate and enhance the understanding of protein function.

\section{Construction and content}

\section{Data sources}

The major challenge for CoBaltDB is to collect and integrate into a centralized open-access reference database, non-redundant subcellular prediction features for complete prokaryotic orfeomes. Our initial dataset contained 784 complete genomes (731 bacteria and 53 Archaea), downloaded with all plasmids and chromosomes (1468 replicons in total), from the NCBI ftp server ftp://ftp. ncbi.nih.gov/genomes/Bacteria in mid-December 2008. This dataset contains 2,548,292 predicted non-redundant proteins (Additional file 1).

The CoBaltDB database was designed to associate results from disconnected resources. It contains three main types of data: $i$ ) CoBaltDB pre-computed prediction using 23 feature-based localization tools (Table 1), ii) CoBaltDB pre-computed prediction obtained using 5 localization meta-tools (Table 2) and iii) data collected from 20 public databases with both predicted and experimentally determined subcellular protein localizations (Table 3).

These data were organized in five "boxes" with regard to the features predicted: three boxes correspond to signal peptide detection (Lipoprotein, Tat- and Sec- dependent targeting signals); one box for the prediction of alpha-transmembrane segments (TM-Box); and one box, only available for diderms (Gram-negatives), for outer membrane localization through prediction of betabarrels. 
Table 1 A summary of CoBaltDB precomputed features-tools

\begin{tabular}{|c|c|c|c|c|c|c|c|}
\hline \multirow{2}{*}{$\begin{array}{l}\text { Program } \\
\text { LipoP } 1.0 \text { Server }\end{array}$} & \multirow{2}{*}{$\begin{array}{l}\text { Reference } \\
{[59]}\end{array}$} & \multirow{2}{*}{$\begin{array}{l}\text { Analytical method } \\
\mathrm{HMM}+\mathrm{NN}\end{array}$} & \multicolumn{4}{|c|}{ CoBaltDB features prediction group(s) } & \\
\hline & & & LIPO & & SEC & & \\
\hline DOLOP & {$[57]$} & $\mathrm{RE}$ & LIPO & & & & \\
\hline LIPO & {$[56]$} & RE & LIPO & & & & \\
\hline TatP 1.0 & [53] & $R E+N N$ & & TAT & & & \\
\hline TATFIND 1.4 & {$[52]$} & RE & & TAT & & & \\
\hline PrediSi & [112] & Position weight matrix & & & SEC & & \\
\hline SignalP 3.0 Server & {$[45-47]$} & $\mathrm{HMM}+\mathrm{NN}$ & & & SEC & & \\
\hline SOSUlsignal & [113] & Multi-programs & & & SEC & & \\
\hline SIG-Pred & J.R. Bradford & Matrix & & & SEC & & \\
\hline RPSP & {$[44]$} & NN & & & SEC & & \\
\hline Phobius & {$[48,49]$} & HMM & & & SEC & $\alpha \mathrm{TMB}$ & \\
\hline НMMTOP & {$[71]$} & HMM & & & & $\alpha \mathrm{TMB}$ & \\
\hline TMHMM Server v.2.0 & [70] & HMM & & & & $\alpha \mathrm{TMB}$ & \\
\hline TM-Finder & {$[65]$} & AA FEATURES & & & & $\alpha \mathrm{TMB}$ & \\
\hline SOSUI & [114] & AA FEATURES & & & & $\alpha \mathrm{TMB}$ & \\
\hline SVMtm & [73] & SVM & & & & $\alpha T M B$ & \\
\hline SPLIT 4.0 Server & [115] & AA FEATURES & & & & $\alpha \mathrm{TMB}$ & \\
\hline MCMBB & [116] & HMM & & & & & $\beta$ Barrel \\
\hline TMBETADISC: & [117] & & & & & & \\
\hline & & AA FEATURES & & & & & $\beta$ Barrel \\
\hline _DIPEF & & Dipeptide composition & & & & & $\beta$ Barrel \\
\hline & & Motif(s) & & & & & $\beta$ Barrel \\
\hline TMB-Hunt2 & [118] & SVM & & & & & $\beta$ Barrel \\
\hline
\end{tabular}

HMM: Hidden Markov Model, NN: Neural Network, RE: Regular Expression, AA: Amino Acid, SVM: Support Vector Machine

Table 2 A summary of CoBaltDB precomputed meta-tools

\begin{tabular}{llll}
\hline Program & Reference & Analytical method & Localizations \\
\hline Subcell Specialization Server 2.5 & {$[119]$} & Multiple classifiers & 5 diderms/3 monoderms \\
SLP-Local & {$[120]$} & SVM & 3 with no distinction \\
SubLoc V1.0 & {$[121]$} & SVM & 3 with no distinction \\
Subcell (Adaboost method) & {$[122]$} & AdaBoost algorithm & 3 with no distinction \\
SOSUIGramN & {$[123]$} & Physico-chemical parameters & 5 diderms/no monoderm \\
\hline
\end{tabular}

SVM: Support Vector Machine

\section{Data generation}

There is a great diversity of web and stand-alone resources for the prediction of protein subcellular location. We retrieved and tested 99 currently (in 2009) available specialized and global tools (software resources) that use various amino acid features and diverse methods: algorithms, HMM, NN, Support Vector Machine (SVM), software suites and others), to predict protein subcellular localization (Additional file 2). All tools were evaluated: some are included in CoBaltDB, some may be launched directly from the platform (Table 4), and others were excluded because of redundancy or processing reasons or both (Table 5). Some tools are specific to Gram-negative or Gram-positive bacteria. Many prediction methods applicable to both Gram categories have different parameters for the two groups of bacteria. For these reasons, each NCBI complete bacterial and archaeal genome implemented in CoBaltDB was registered as "monoderm" or "diderm", on the basis of information in the literature and phylogeny (Additional file 3). Monoderms and diderms were considered as Gram-negative and Gram-positive, respectively. All archaea were classified as monoderm prokaryotes since their cells are bounded by a single cell membrane and possess a cell envelope [3,95]. An exception was made for Ignicoccus hospitalis as it owns an outer sheath resembling the outer membrane of gramnegative bacteria [96].

Currently, CoBaltDB contains pre-computed results obtained with 48 tools and databases, and additionally provides pre-filled access to 50 publicly available tools that could not be pre-computed or that provide new 
Table 3 A summary of CoBaltDB integrated databases and tools features.

\begin{tabular}{|c|c|c|c|c|}
\hline Databases & Reference & Features predicted & Genome(s) & Protein numbers \\
\hline EchoLOCATION & {$[124]$} & Subcellular-location (EXP) & E. coli K-12 & 4330 (506 exp) \\
\hline Ecce & - & Subcellular-location & E. coli K-12 & 306 \\
\hline LocateP DataBase & [89] & Subcellular-location & $178 \mathrm{MD}$ & 542788 \\
\hline cPSORTdb & [91] & Subcellular-location & $140 \mathrm{BA}$ & 1634278 \\
\hline ePSORTdb & [91] & Subcellular-location (EXP) & & 2165 \\
\hline THGS & [125] & Transmembrane Helices & 689 PROK & 465411 \\
\hline Augur & {$[88]$} & Subcellular-location & $126 \mathrm{MD}$ & 111223 \\
\hline CW-PRED & [126] & Cell-anchored (surface) & $94 \mathrm{MD}$ & 954 \\
\hline PROFtmb & {$[78]$} & Beta-barrel (OM) & $78 \mathrm{DD} / 19 \mathrm{MD}$ & 2152 \\
\hline HHomp & [127] & Beta-barrel (OM) & & 12495 \\
\hline PRED-LIPO & {$[58]$} & Lipoprotein SPs & $179 \mathrm{MD}$ & 895 \\
\hline $\mathrm{SPdb}$ & {$[90]$} & Signal peptides (SPs) & 855 PROK & 7062 \\
\hline ExProt & {$[128]$} & Signal peptides (SPs) & $23 \mathrm{AR} / 61 \mathrm{MD} / 115 \mathrm{DD}$ & \\
\hline Signal Peptide Website & - & Signal peptides (SPs) & $384 \mathrm{BA}$ & 1161 (EXP) \\
\hline PRED-SIGNAL & [129] & Signal peptides (SPs) & $48 \mathrm{AR}$ & 9437 \\
\hline TMPDB & [130] & Alpha Helices \& Beta-barrel & & 188 \\
\hline DTTSS & Shandong Univ. & Type III secretion system & & 1035 \\
\hline TOPDB & {$[131]$} & Transmembrane Proteins & $755 \mathrm{BA} / 16 \mathrm{AR}$ & \\
\hline TMBC-Database & Andrew Garrow & Transmembrane Beta-barrel & & 1219 \\
\hline Swissprot signal testset & {$[132]$} & Signal peptides (SPs) (EXP) & $176 \mathrm{SP}+/ 122 \mathrm{SP}-$ & \\
\hline
\end{tabular}

$\mathrm{MD}=$ monoderm, $\mathrm{DD}=$ diderm. AR (Archeae), BA (Bacteria), PROK (Prokaryotes) include both bacteria and Archaee, EXP = Experimental database

information (tools dedicated to a special phylum, consensus tools or tools predicting proteins secreted via other pathways). The data pre-computing process is illustrated in Figure 1; web-based and stand-alone tools were used separately. Web-based localization prediction tools were requested via a Web automat, a python automatic submission workflow using both "httplib" and "urllib" libraries. A different script was created for each tool. For web-tools with no equivalent (such as "TatP" for Tat-BOX and "LIPO" for Lipoprotein-BOX) and incompatible with automatic requests, we collected results manually. CoBaltDB also provides a platform with automatically pre-filled forms for additional submissions to a selection of fifty recent or specific web tools (Table 4). The stand-alone tools were installed on a Unix platform (unique common compatible platform) and included in a global python pipeline with the HTTP request scripts. We selected information from a up-todate collection of 20 databases and integrated this data within CoBaltDB; these databases were retrieved by simple downloading or creating an appropriate script which navigates on the web databases to collect all protein information. The global python pipeline used multithreading to speed up the pre-computation of the 784 proteomes.

\section{Database Creation and Architecture}

For each protein, every output collected (a HTML page for web tools and a text file for standalone applications) was parsed and selected items were stored in a particular format: binary "marshal" files. The object structure obtained by parsing tool output was directly saved into a marshal file, allowing a quick and easy opening by directly restoring the initial parsing object. Another script then creates the CoBaltDB repository, by reading and analysing all marshal files to generate a specific formatted file (".cbt") for each replicon. These files contain all the required protein information and a simplified representation of the tools' results. Some initialization files containing information about phylogeny or genome features are also used.

The repository is used by the Graphical User Interface (GUI) to display CoBaltDB information. For raw data from tools, the GUI accesses the marshal file directory.

\section{Accessing the CoBaltDB Repository and Raw Data}

The CoBaltDB platform has been developed as a clientserver application. The server is installed at the Genouest Bioinformatics platform http://www.genouest.org/? lang=en. The client is a Java application that needs to be locally downloaded by the users. Queries are submitted to the server-side CoBaltDB repository using a locally installed client GUI that provides tabular and graphical representations of the data. The repository is accessed through SOAP-based web services (Simple Object Access Protocol), implemented in Java 5 using the Apache Axis 1.4 toolkit and deployed on the servlet engine Tomcat 5.5.20. CoBaltDB integrates: an 
Table 4 Tools available using CoBaltDB "post" window

\begin{tabular}{|c|c|c|c|c|c|c|}
\hline Program & Reference & Analytical method & CoBaltDB fe & tures prediction group(s) & & \\
\hline LipPred & [133] & Naive Bayesian Network & LIPO & & & \\
\hline PRED-LIPO & {$[58]$} & $\mathrm{HMM}$ & LIPO & & (only Monoderm) & \\
\hline SPEPLip & [134] & NN & LIPO & SEC & & \\
\hline SecretomeP & [135] & Pattern \& NN & & $\triangle S E C \_S P$ & & \\
\hline Signal-3L & [136] & Multi-modules & & SEC & & \\
\hline Signal-CF & [137] & Multi-modules & & SEC & & \\
\hline Signal-Blast & [138] & BlastP & & SEC & & \\
\hline Sigcleave & EMBOSS & Von Heijne method & & SEC & & \\
\hline PRED-SIGNAL & [129] & $\mathrm{HMM}$ & & SEC & (only Archae) & \\
\hline Flafind & [139] & AA features & & T3SS Archae + T4SS Bacteria & & \\
\hline T3SS_prediction & [110] & SVM \& NN & & T3SS & & \\
\hline EffectiveT3 & [111] & Machine learning & & T3SS & & \\
\hline NtraC Signal Analysis & {$[140]$} & Pattern model & & SEC (long SP) & & \\
\hline Philius & {$[141]$} & HMM & & SEC & $\alpha T M B$ & \\
\hline (SP)OCTOPUS & {$[142,143]$} & Blast Homology, NN, HMM & & SEC & $\alpha T M B$ & \\
\hline MemBrain & {$[144]$} & Machine learning & & SEC & $\alpha T M B$ & \\
\hline DAS & [145] & Dense Alignment Surface & & & $\alpha \mathrm{TMB}$ & \\
\hline HMM-TM & {$[146]$} & HMM & & & $\alpha T M B$ & \\
\hline SVMtop Server 1.0 & [147] & SVM & & & $\alpha T M B$ & \\
\hline UMDHMM_TMHP & {$[148]$} & HMM & & & $\alpha T M B$ & \\
\hline waveTM & [149] & Hydropathy signals algorithm & & & $\alpha T M B$ & \\
\hline PRED-TMR & {$[150]$} & AA features & & & $\alpha \mathrm{TMB}$ & \\
\hline TMAP & [67] & AA features & & & $\alpha T M B$ & \\
\hline igTM & [151] & Grammatical Inference & & & $\alpha T M B$ & \\
\hline TOPCONS & {$[152]$} & Tools Consensus & & & $\alpha T M B$ & \\
\hline TUPS & [153] & Tools Consensus & & & $\alpha T M B$ & \\
\hline ConPred II & {$[154]$} & Tools Consensus & & & $\alpha \mathrm{TMB}$ & \\
\hline MEMSAT3 & {$[66,155]$} & NN & & & $\alpha T M B$ & \\
\hline SABLE & [156] & NN & & & $\alpha T M B$ & \\
\hline TM-Pro & {$[64,157]$} & AA features & & & $\alpha \mathrm{TMB}$ & \\
\hline ProspRef & - & Knowledge-based method & & & $\alpha \mathrm{TMB}$ & \\
\hline PSIPRED & {$[158,159]$} & NN, PSSM & & & $\alpha T M B$ & \\
\hline NPS@ & {$[160]$} & Tools Consensus & & & $\alpha \mathrm{TMB}$ & \\
\hline SAM-T08 & [161] & $\mathrm{HMM}$ & & & $\alpha T M B$ & \\
\hline PORTER & [162] & NN & & & $\alpha T M B$ & \\
\hline TMPred & EMBnet & Weight-matrices & & & $\alpha T M B$ & \\
\hline TMMOD & {$[163]$} & HMM & & & $\alpha T M B$ & \\
\hline TopPred ॥ & [61] & G. von Heijne algorithm & & & $\alpha T M B$ & \\
\hline YASPIN & {$[164]$} & Hidden Neural Network & & & $\alpha T M B$ & \\
\hline MemType-2L & [165] & PseudoPSSM, classifier & & & Membrane Type & \\
\hline BOMP & {$[84]$} & AA features & & & & $\beta$ Barrel \\
\hline TMBETADISC-RBF & [87] & RBF network, PSSM & & & & $\beta$ Barrel \\
\hline TMBETA-NET & {$[117]$} & AA features & & & & $\beta$ Barrel \\
\hline PRED-TMBB & {$[85]$} & HMM & & & & $\beta$ Barrel \\
\hline ConBBPred & {$[76]$} & Tools Consensus & & & & $\beta$ Barrel \\
\hline CW-PRED (submit) & {$[126]$} & HMM & & Cell-Wall (only Monoderm) & & \\
\hline ProtCompB & SoftBerry & Multi-methods & Localization & & & \\
\hline CELLO & {$[166]$} & SVM & Localization & & & \\
\hline PSL101 & [167] & SVM, structure homology & Localization & & & \\
\hline PSLpred & [168] & SVM & Localization & & & \\
\hline GPLoc-neg & [169] & Basic classifier & Localization & & (only Diderm) & \\
\hline
\end{tabular}


Table 4: Tools available using CoBaltDB "post" window (Continued)

\begin{tabular}{llll}
\hline GPLoc-pos & {$[170]$} & Basic classifier & Localization \\
LOCtree & {$[171]$} & SVM & Localization \\
PSORTb & {$[91]$} & Multi-modules & Localization \\
SLPS & {$[172]$} & Nearest Neighbor on domain & Localization \\
Couple-subloc v1.0 & Jian Guo & AA features & Localization \\
TBPRED & {$[173]$} & SVM & Localization
\end{tabular}

HMM: Hidden Markov Model, NN: Neural Network, AA: Amino Acid, SVM: Support Vector Machine, PSSM: Position Specific Scoring Matrix, T3SS: Type III Secretion System, RBF: Radial Basis Function

Table 5 Tools and Database not available in CoBaltDB

\begin{tabular}{|c|c|c|c|c|c|}
\hline Program & Reference & Analytical method & CoBaltDB features $\mathrm{pr}$ & tion group(s) & \\
\hline SpLip & {$[174]$} & Weight matrix & LIPO & (only Spirochaetal) & \\
\hline PROTEUS2 & {$[175]$} & Multi-Methods & SEC & $\alpha \mathrm{TMB}$ & $\beta$ Barre \\
\hline PRED-TMR2 & {$[176]$} & NN & & $\alpha T M B$ & \\
\hline PRODIV-TMHMM & [72] & Multi HMM & & $\alpha T M B$ & \\
\hline S_TMHMM & [72] & $\mathrm{HMM}$ & & $\alpha T M B$ & \\
\hline TransMem & {$[69]$} & NN & & $\alpha T M B$ & \\
\hline BPROMPT & {$[177]$} & Bayesian Belief Network & & $\alpha T M B$ & \\
\hline orienTM & {$[178]$} & Statistical analysis & & $\alpha \mathrm{TMB}$ & \\
\hline APSSP2 & [179] & Multi-Methods & & Secondary structure & \\
\hline PRALINE_TM & {$[180]$} & Alignment, tools consensus & & Secondary structure & \\
\hline OPM (DB) & [181] & Multi-Methods & & Membrane orientation & \\
\hline MP_Topo (DB) & [182] & Experimental & & $\mathrm{TMB}$ & \\
\hline PDBTM (DB) & [183] & TMDET algorithm & & $\mathrm{TMB}$ & \\
\hline TMB-HMM & A.Garrow & HMM, SVM & & & $\beta$ Barre \\
\hline TMBETA-SVM & {$[86]$} & SVM & & & $\beta$ Barre \\
\hline TMBETA-GENOME (DB) & [184] & Multi-Methods & & & $\beta$ Barre \\
\hline PredictProtein & [185] & Alignment, Multi-Methods & Localization & & \\
\hline EcoProDB (DB) & [186] & Identification on 2D gels & Localization & (only E.coli) & \\
\hline LOCTARGET (DB) & [187] & Multi-Methods & Localization & & \\
\hline DBMLoc (DB) & [188] & _ & Localization & & \\
\hline
\end{tabular}

NN: Neural Network, HMM: Hidden Markov Model, SVM: Support Vector Machine

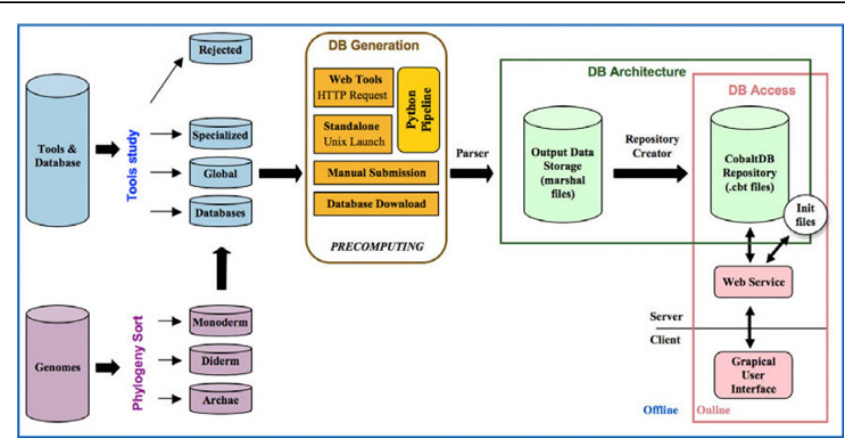

Figure 1 A schematic view of the CoBaltDB workflow. CoBaltDB integrates the results of 43 localization predictors for 784 complete bacterial and archaeal proteomes. Each complete NCBI prokaryotic genome implemented in CoBaltDB was classified as: archaea, or monoderm or diderm bacteria. 101 protein subcellular location predictors were evaluated and few were rejected. Selected tools were classified as: feature localization tools (Specialized), localization meta-tools (Global) or databases. The data recovery process was performed manually or via a Web automat using a python automatic submission workflow for both stand-alone and web-based tools. Databases were downloaded. For each protein, ouptuts collected were parsed and selected items were stored in particular CoBaltDB formatted files (.cbt). The parsing pipeline creates one ".cbt" file per replicon to compose the final CoBaltDB repository. The client CoBaltDB Graphical User Interface communicates with the server-side repository via web services to provide graphical and tabular representations of the results. 
initialization web service (that returns the current list of genomes supported); two repository web services that allow querying the database either by specifying a replicon or a list of locus tags; and a raw data web service that retrieves all recorded raw data generated by a given tool for the specified locus tag.

\section{Utility}

\section{Running CoBaltDB}

Our goal was to build an open-access reference database providing access to protein localization predictions. CoBaltDB was designed to centralize different types of data and to interface them so as to help researchers rapidly analyse and develop hypotheses concerning the subcellular distribution of particular protein(s) or a given proteome. This data management allows comparative evaluation of the output of each tool and database and thus straightforward identification of inaccurate or conflicting predictions.

We developed a user-friendly CoBaltDB GUI as a Java 5 client application using NetBeans 5.5.1 IDE. It presents four tabs that perform specific tasks: the "input" tab (Figure 2) allows selecting the organism whose proteome localizations will be presented, using organism name completion or through an alphabetical list. Alternatively, users may also enter a subset of proteins, specified by their locus tags. The "Specialized tools" tab (Figure 3) supplies a table showing, for each protein identified by its locus tag or protein identifier, some annotation information such as its gene name, description and links to the corresponding NCBI and KEGG web pages. Clicking on a "locus tag" opens a navigator window with the related KEGG link, and clicking on a "protein Id" opens the corresponding NCBI entry web page. The table shows, for each protein and for each feature box (Tat, Sec, Lipo, $\alpha$ TMB, $\beta$ Barrel), a heat map (white/blue) representing the percentage of tools predicting the truth/presence of the corresponding localization feature in the protein considered. Clicking on the heat map opens a new window that shows the raw data generated by each tool of the considered feature box, thus allowing the investigator to access the tool-specific information they are used to. The predictions of related feature databases are given next to the corresponding heat-map. The proteins which are referred to by the databases implemented in CobaltDB as having an experimentally determined localization appear with a yellow background colour. This representation enables the user to observe graphically the distribution of tools predicting each type of feature. The "meta-tools" tab (Figure 4) provides the predictions given by multi-modular prediction software (meta-tools or global databases) that use various techniques to predict directly three to five subcellular protein localizations in mono- and/or diderm bacteria (Table 4). The descriptions of the localizations were standardised to ease interpretation by the

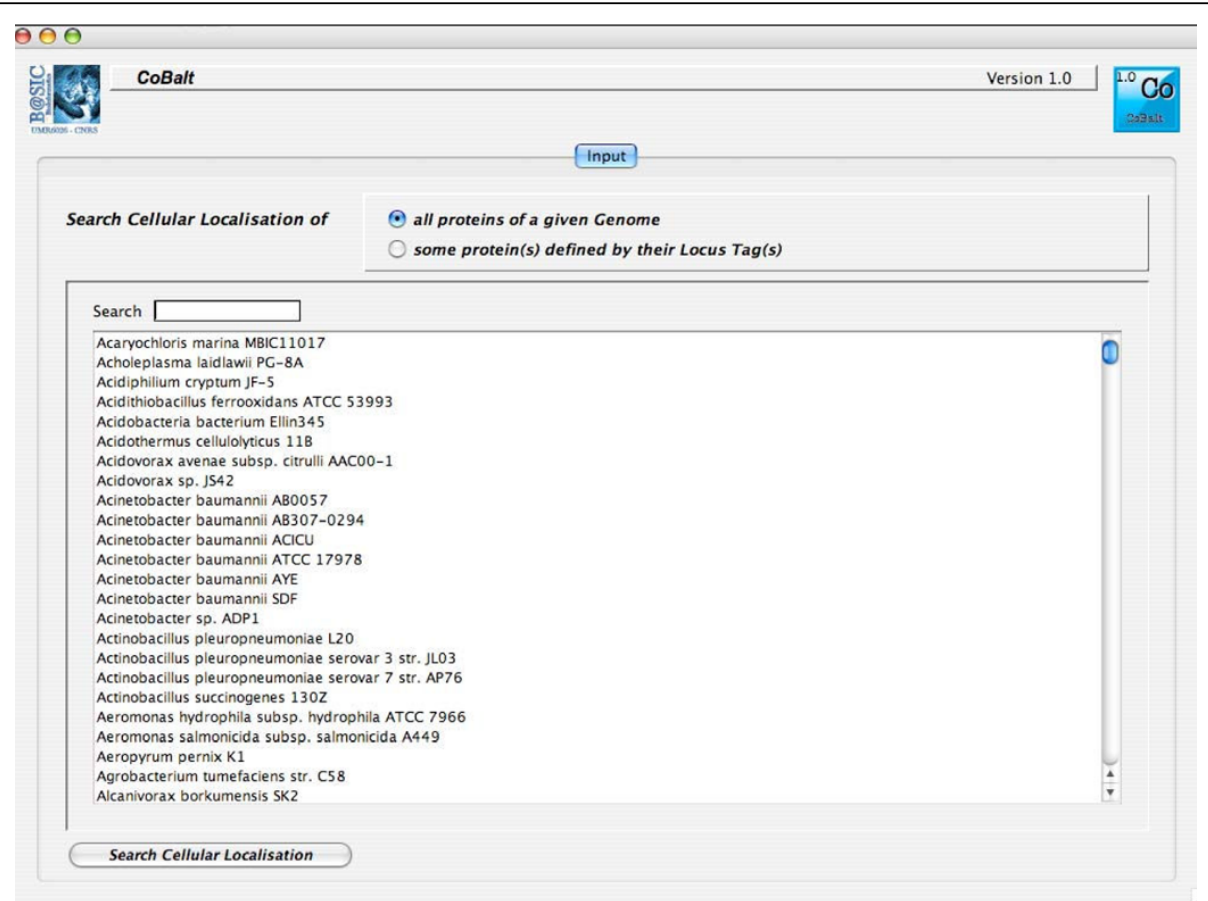

Figure 2 A snapshot of the CoBaltDB input interface. The "input" module allows the selection of organisms, using organism name completion or through an alphabetical list. Users can also enter a subset of proteins, specified by their locus tags. 


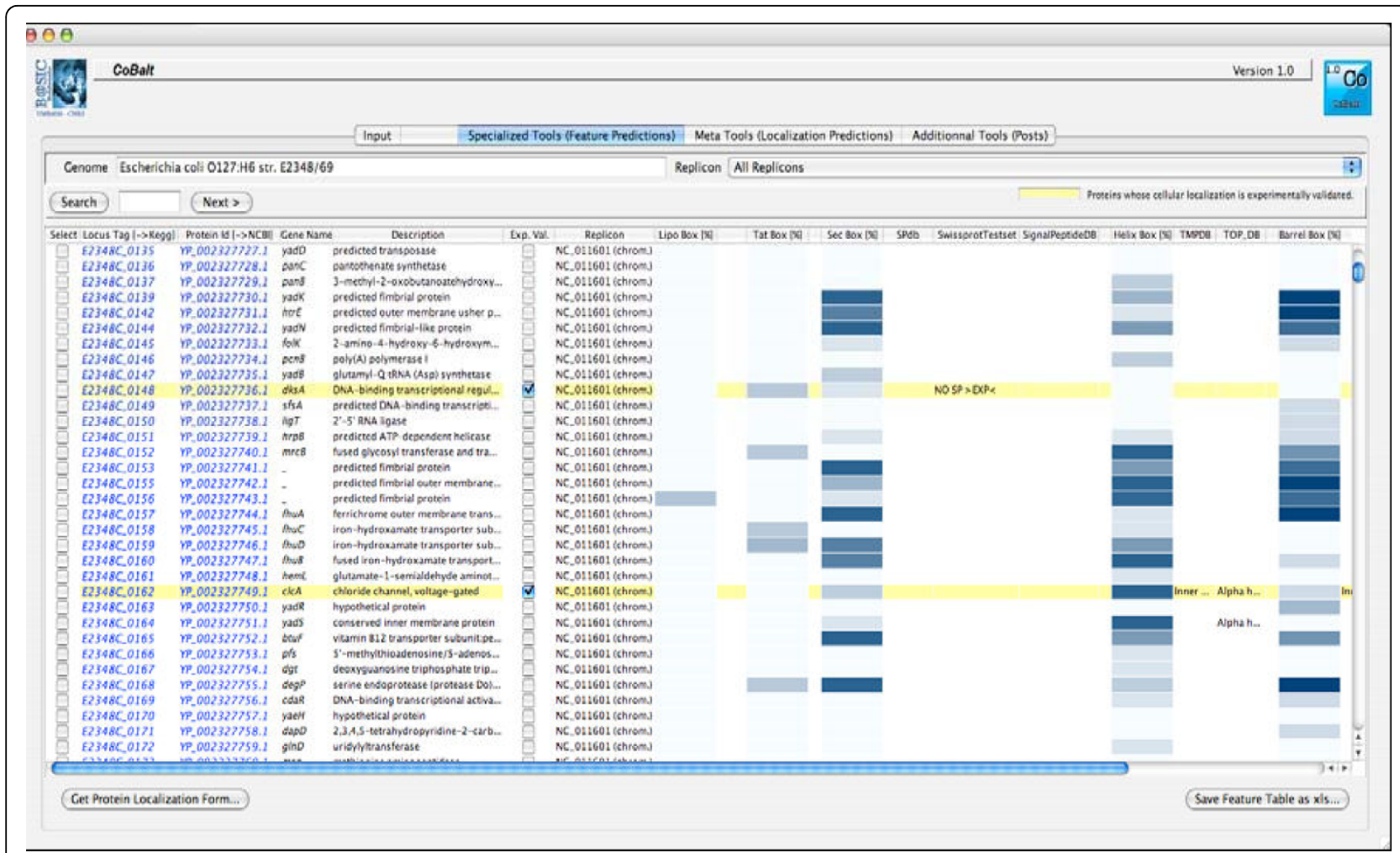

Figure 3 The CoBaltDB Specialized Tools viewer. The "Specialized tools" browser supplies a tabular output for every protein, enriched with the protein's annotation including locus tag, protein identifier, gene name (if available) and product descriptions. Clicking on each "locus tag" opens a navigator window with related KEGG link whereas clicking on every "protein Id" opens the corresponding NCBI entry web page. Clicking on the white/blue heat map reveals the raw results of all tools corresponding to the feature box considered.

investigator. Both tables may be searched for occurrences of any string of characters via the search button, facilitating retrieval of a particular locus tag, protein id, accession number or even a gene name or annotation description. Both tables may be sorted with respect to any column, i.e. in alphanumerical order for the locus tags, protein identifiers, annotation descriptions and localization predictions, or in numerical order for the percentages. This makes it straightforward to identify all proteins with particular combinations of localization features. Both tables may be saved as Excel files. Finally, the CoBaltDB "additional tools" tab (Figure 5) enables queries to be submitted to a set of 50 additional tools by pre-filling the selected forms with the selected protein sequence and Gram information whenever appropriate. For this use, the investigator might have to enter additional parameters.

Finally, for each protein, all results were summarized in a synopsis (Figure 6); the synopsis presents the results generated by all the tools in a unified manner, and includes a summary of all predicted cleavage sites and membrane domains. This "standardized" form thus provides all relevant information and lets the investigators establish their own hypotheses and conclusions. This form may be saved as a .pdf file (Figure 6). Examples of using the CoBaltDB synopsis are provided below in the second case study.

\section{Selected CoBaltDB uses}

We propose to illustrate briefly some possible uses of CoBaltDB.

\section{1-Using CoBaltDB to compare subcellular prediction tools} and databases

The various bioinformatic approaches developed for computational determination of protein subcellular localization exhibit differences in sensitivity and specificity; these differences are mainly the consequences of the types of sequences used as training models (diderms, monoderms, Archaea) and of the methods applied (regular expressions, machine learning or others). By interfacing the results from most of the reliable predictions tools, CoBaltDB provides immediate comparisons and constitutes an accurate and high-performance resource to identify and characterize candidate "non-cytoplasmic" proteins. As an example, using CoBaltDB to analyse the 82 proteins that compose the experimentally confirmed 


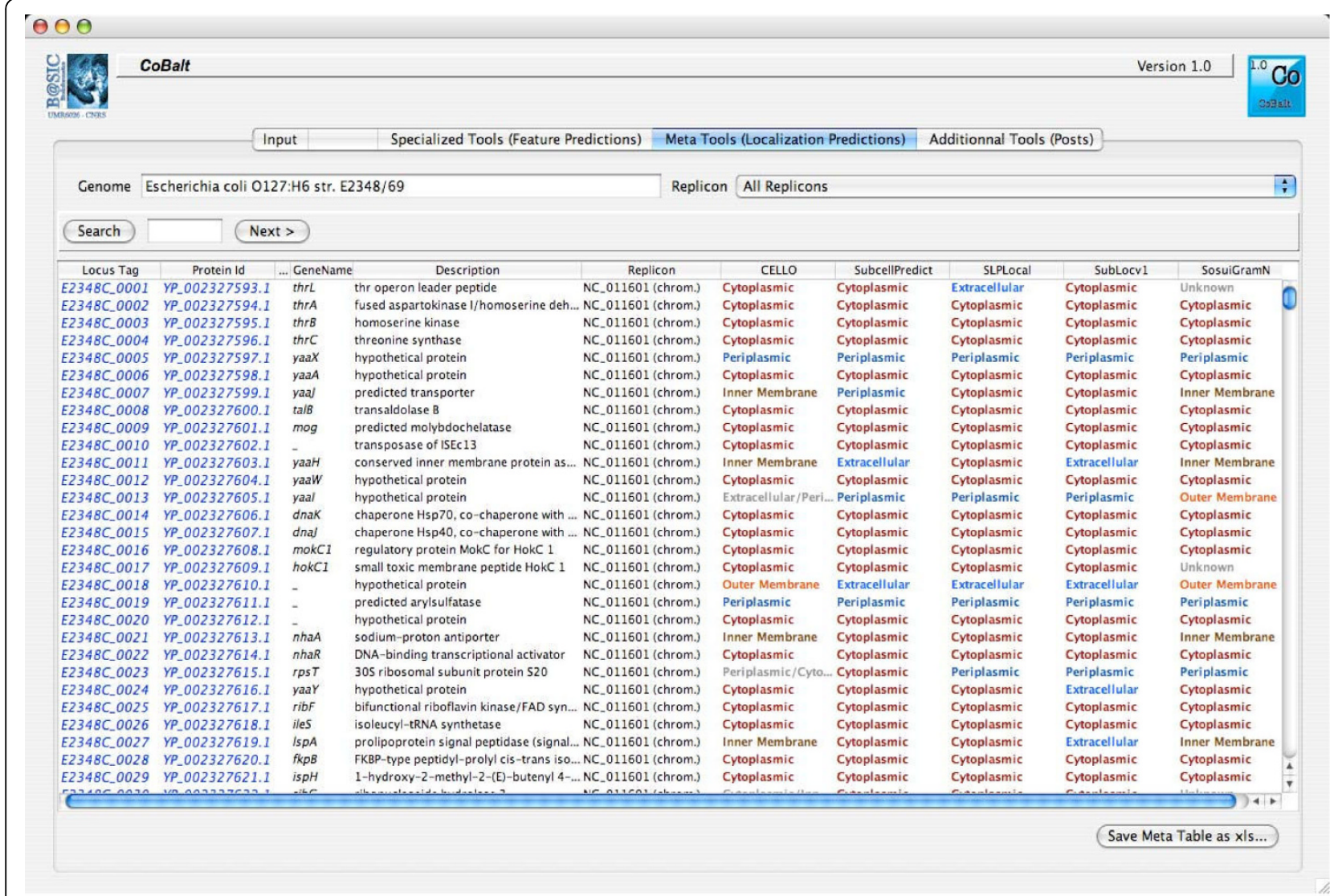

Figure 4 The CoBaltDB Meta-Tools interface. The "meta-tools" panel presents the CoBaltDB-computed results for multi-modular prediction software that uses various techniques to directly predict 3 to 5 subcellular localizations for proteins in mono- and/or diderm bacteria.

"Lipoproteome" of E. coli K-12 [97] shows that 72 are correctly predicted by the three precomputed tools (LipoP [59], DOLOP [57] and LIPO [56]), and that the other 10 are only identified by two of the three tools (Additional file 4A). Eight of these lipoproteins were not detected by DOLOP, because the regular expression pattern allowing detection of the lipidation sequence ([LVI] [ASTVI] [GAS] [C] lipobox) is too stringent (Additional file 4B). By comparison, the PROSITE lipobox pattern (PS00013/PDOC00013) is more permissive ([DERK](6)[LIVMFWSTAG] (2)- [LIVMFYSTAGCQ]- [AGS]-C). This example demonstrates that using a single tool may result in errors and suggests that the best approach is to combine the various "features-based" methods available and compare their findings. This view also applies to meta-tools predictors. E. coli $\mathrm{K} 12$ lipoproteins can be found anchored to the inner or the outer membrane through attached lipid, but some of them are periplasmic (Additional file 4A). The comparison of in silico subcellular localization assignments with experimental findings clearly indicates that all meta-tools require significant improvements in accuracy and precision, that none should be used to the exclusion of the others. It also appears that analysis with specialized tools, organized on a "one feature at a time" basis (Lipo SPs, TAT SPs ...), most reliably gives predictions consistent with experimental data. For this purpose, CoBaltDB is a unique and innovative resource.

\section{2-Using CoBaltDB to analyse protein(s) and a proteome}

One valuable property of CoBaldDB is to recapitulate all pre-computed predictions in a unique A4-formated synopsis. This summary is very helpful for assessing computational data such as the variation and frequency in the predictions of signal peptide cleavage sites: such predictions are sometimes significantly consistent, but often are not in agreement with each other (Figure 7A). However, correct identification of signal peptide cleavage sites is essential in many situations, especially for producing secreted recombinant proteins.

The CoBaltDB synopsis could also be used to discriminate between SignalPeptidaseII- and SignalPeptidaseI-cleaved signals and between SPs and N-terminal transmembrane helices. Indeed, most localization predictors have difficulties distinguishing between type I 


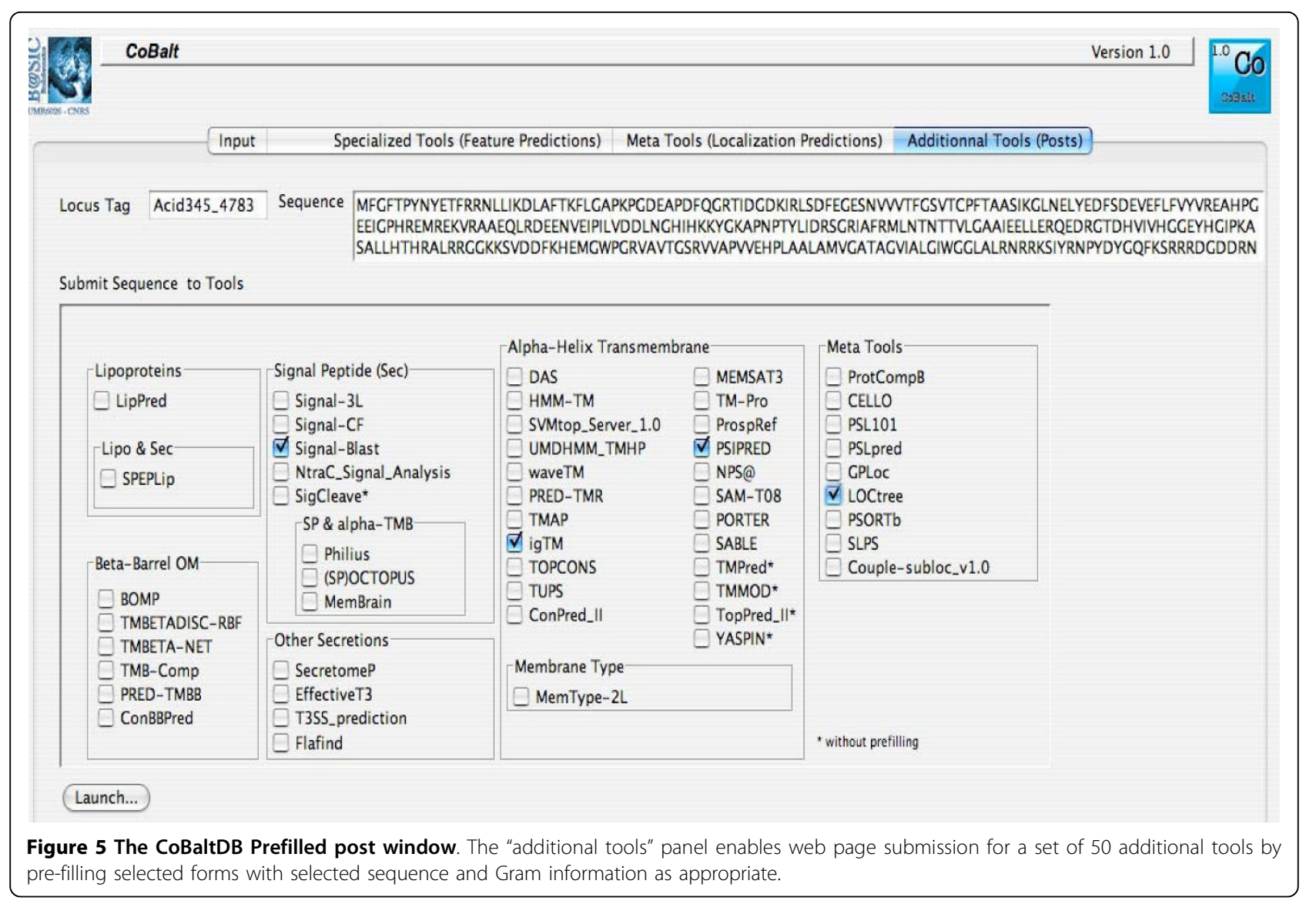

and type II signal peptidase cleavages. CoBaltDB can be exploited in an interesting way to benchmark this prediction by displaying all cleavage site predictions in a "decreasing sensitivity" arrangement (SpII then Tatdependant SPI then Sec-SPI). By considering lipoprotein datasets from different organisms, we evidenced two principal profiles (Figure 7B) and found that all experimentally validated lipoproteins score $100 \%$ (all tools give the same prediction) or $66 \%$ in the CoBaltDB LIPO column (see explanation in the paragraph above). In addition, in almost all of the examined cases, tools dedicated to Twin-arginine SP detection do not identify SpII-dependent SP, whereas the Sec-SP predictors detect both Sec and Tat-type I as well as type II signal-anchor sequences.

These observations allow us to propose, for our data set, thresholds for each box: as previously illustrated, lipoproteins have score $>66 \%$ in the LIPO prediction box; Tat-secreted proteins have $0 \%$ in the LIPO box and $100 \%$ for the two TAT-dedicated tools; Sec-secreted proteins have $33 \%$ in the LIPO Box (due to the fact that LipoP detects both SpI and SpII [59]), 0\% in the TATtools, and $>80 \%$ in SEC-specialized tools. Rules of this type can be used to check entire proteomes for evaluation of the different secretomes as illustrated in the following case studies.

\section{3-Using CoBaltDB to compare proteomes}

Using CoBaltDB and the thresholds described above, we can compare the predicted lipoproteomes (Figure 8A) of the three completely sequenced substrains of $E$. coli K12: MG1655 and W3110 (both derived from W1485 approximately 40 years ago [98]), and DH10B which was constructed by a series of genetic manipulations [99]. Each of these three substrains encode 89 lipoproteins found in both other substrains (Additional file 4). Four additional lipoproteins are detected in DH10B (BorD, CusC, RlpA and RzoD) and are second copies lipoprotein genes, present in the 113-kb tandemly repeated region of the chromosome (Figure $8 \mathrm{~B}$, coordinates 514341 to 627601 , [99]), and strain DH10B contains one gene encoding the Rz1 proline-rich lipoprotein from bacteriophage lambda absent from the two other substrains. Lipoprotein YghJ, that shares 64\% homology with $V$. cholerae virulence-associated accessory colonization factor AcfD [100], is absent from the DH10B genome annotation. However, comparative genomic analysis 


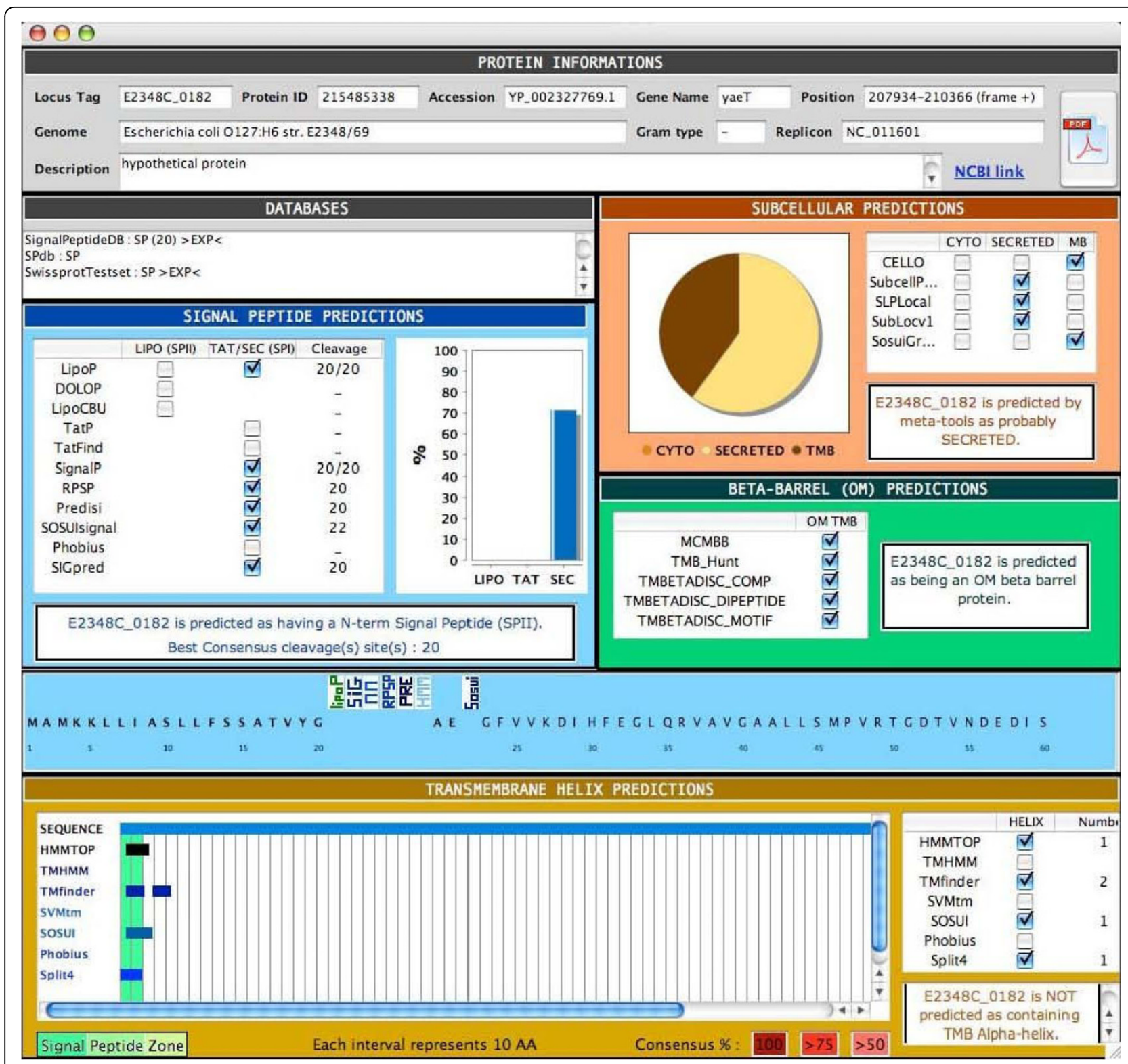

Figure 6 CoBaltDB Synopsis. For any given protein, all results are summarized in a synopsis which presents, in a unified manner, a summary of all predicted cleavage sites and membrane domains. This synopsis can be stored as a .pdf file.

shows that a $y g h J$ locus could be annotated in this strain but corresponds to a pseudogene caused by a frameshift event (Figure 8C). YfbK was also overlooked in the DH10B annotation process but in this case, the gene is intact. Finally, differences between lipoprotein prediction results concerning YafY, YfiM and YmbA are due to erroneous $\mathrm{N}$-terminus predictions. YafY in DH10B was predicted to be a lipoprotein due to the $\mathrm{N}$-terminal 17 aa-long type II signal peptide and was published as a new inner membrane lipoprotein [101]. In substrains MG1655 and WS3110, the original annotation fused the yaf $Y$ loci with its upstream pseudogene $y k f K(137 \mathrm{~N}$ - terminal aa longer). The presumed start codons of YfiM and YmbA in MG1655 were recently changed by adding 17 (lrilfvcsllllsgcsh) and $5(m k k w l) \mathrm{N}$-terminal amino acids, respectively (PMC1325200). These modifications substantially affect the prediction of their subcellular localization. Inspection of the genomic sequences of the two other substrains leads to equivalent changes such that YfiM and YmbA in all three substrains are now predicted to be lipoproteins. In conclusion, using CoBaltDB to compare lipoproteomes between substrains, we were able to detect genomic events as well as "annotation" errors. After correction, we can conclude 


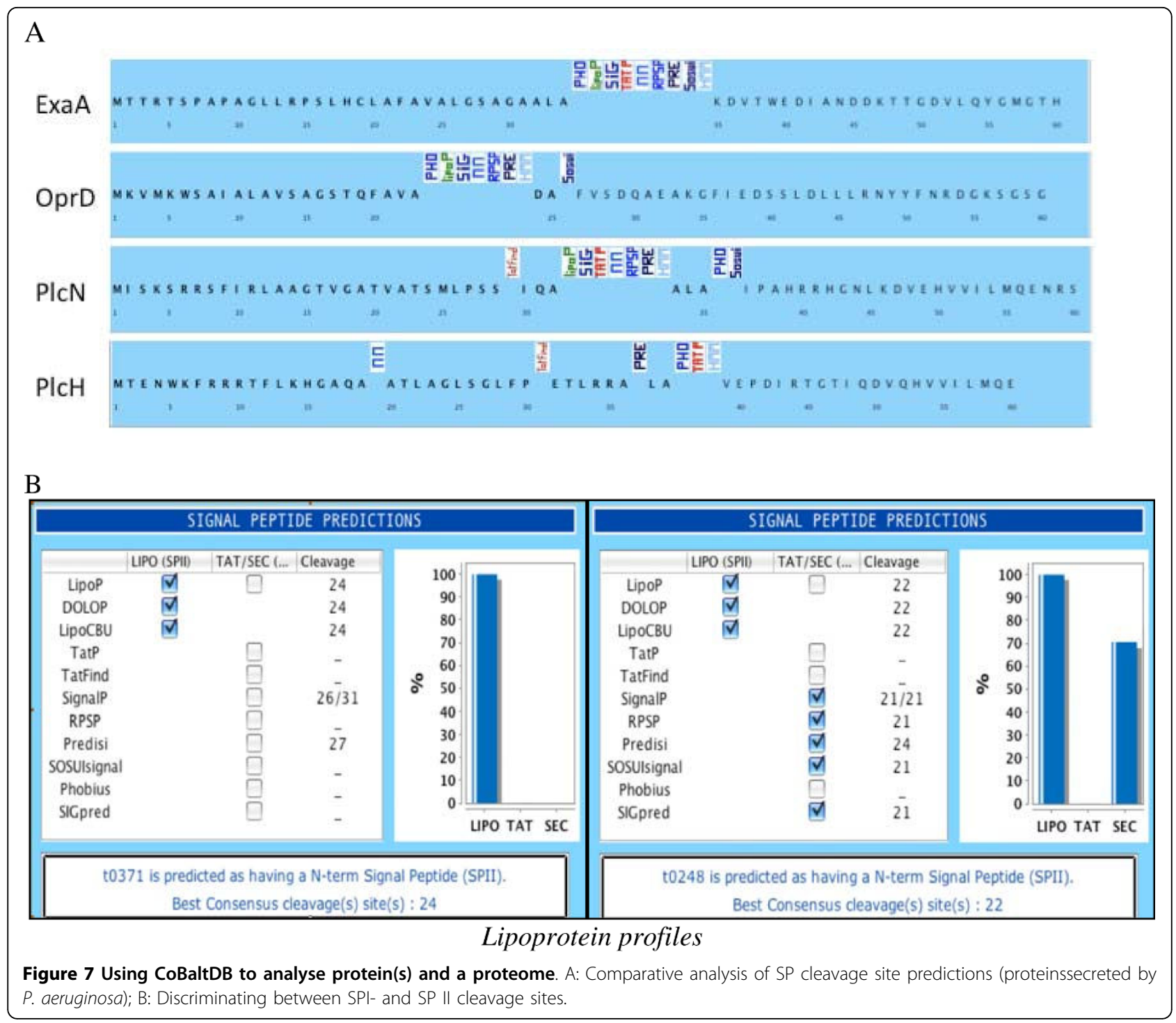

that the three E. coli K12 substrains have 93 lipoproteins in common; that one locus whose function is related to virulence has been transformed into a pseudogene in DH10B; and that DH10B contains five additional lipoproteins due to duplication events and to the presence of prophages absent from the other two substrains (Figure 8D).

\section{4-Using CoBaltDB to improve the classification of orthologous and paralogous proteins}

Protein function is generally related to its subcellular compartment, so orthologous proteins are expected, in most cases, to be in the same subcellular location. Consequently, inconsistencies of location predictions between orthologs potentially indicate distinct functional subclasses. Thus, CobaltDB can be used to help improve the functional annotation of orthologous proteins by adding the subcellular localization dimension. As an example, OxyGene, an anchor-based database of the ROS-RNS (Reactive Oxygen-Nitrogen species) detoxification subsystems for 664 complete bacterial and archaeal genomes, includes 37 detoxicifation enzyme subclasses [102]. Analysis of CoBaltDB subcellular localization information suggested the existence of additional subclasses. For example, 1-cystein peroxiredoxin, PRX_BCPs (bacterioferritin comigratory protein homologs), can be sub-divided into two new subclasses by distinguishing the secreted from the non-secreted forms (Figure 9a). Differences in the location between orthologous proteins are suggestive of functional diversity, and this is important for predictions of phenotype from the genotype.

CoBaltDB is a very useful tool for the comparison of paralogous proteins. For example, quantitative and qualitative analysis of superoxide anion detoxification 


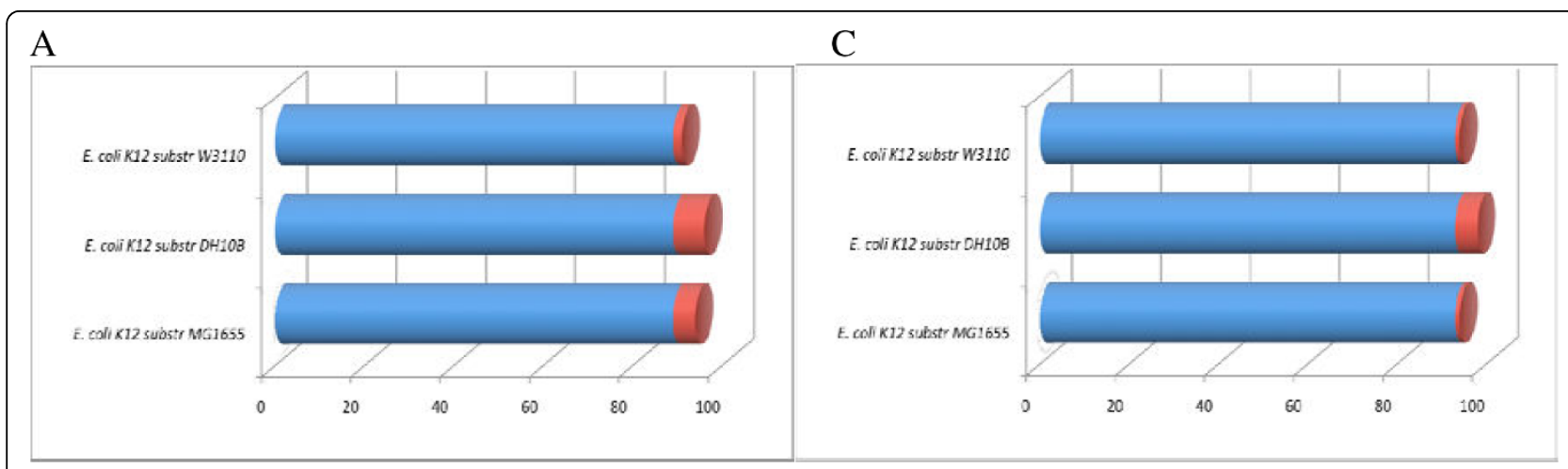

B

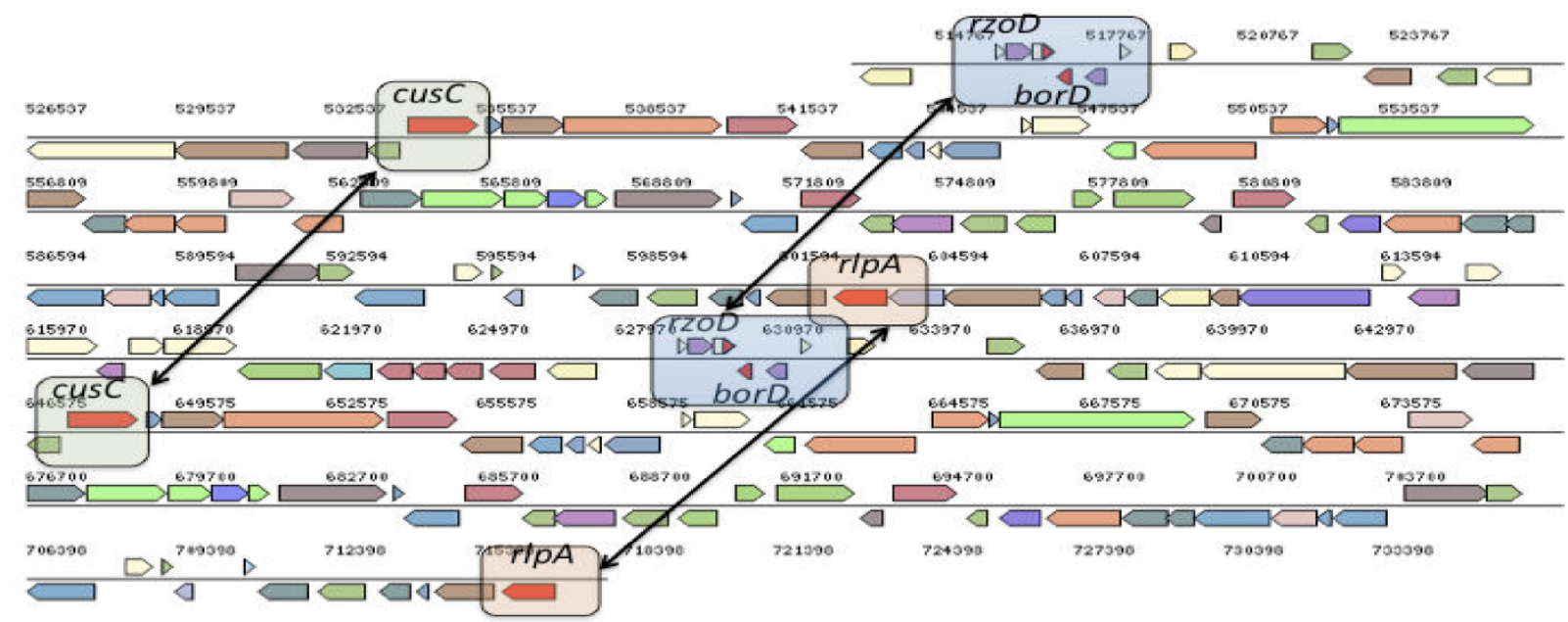

D

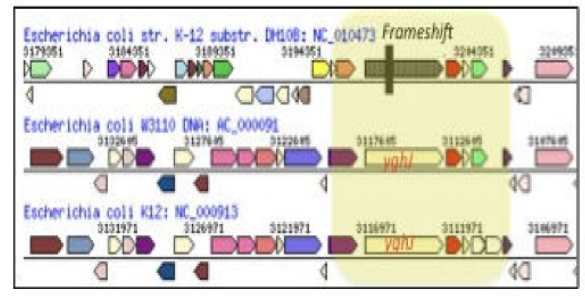

Figure 8 Using CoBaltDB in comparative proteomics. Example of E. coli K12 substrains lipoproteomes.

subsystems using the OxyGene platform identified three iron-manganese Superoxide dismutase (SOD_FMN) in Agrobacterium tumefaciens but only one SOD_FMN and one copper-zinc SOD (SOD_CUZ) in Sinorhizobium meliloti. The number of paralogs and the class of orthologs thus differ between these two closely related genus. However, adding the subcellular localization dimension reveals that both species have machinery to detoxify superoxide anions in both the periplasm and cytoplasm: both one of the three SOD_FMN of $A$. tumefaciens and the SOD_CUZ of S. meliloti are secreted (Figure 9b). CoBaltDB thus helps explain the difference suggested by
OxyGene with respect to the ability of the two species to detoxify superoxide.

\section{Discussion}

CobaltDB allows biologists to improve their prediction of the subcellular localization of a protein by letting them compare the results of tools based on different methods and bringing complementary information. To facilitate the correct interpretation of the results, biologists have to keep in mind the limitations of the tools especially regarding the methodological strategies employed and the training sets used [93]. For example, most specialized tools 


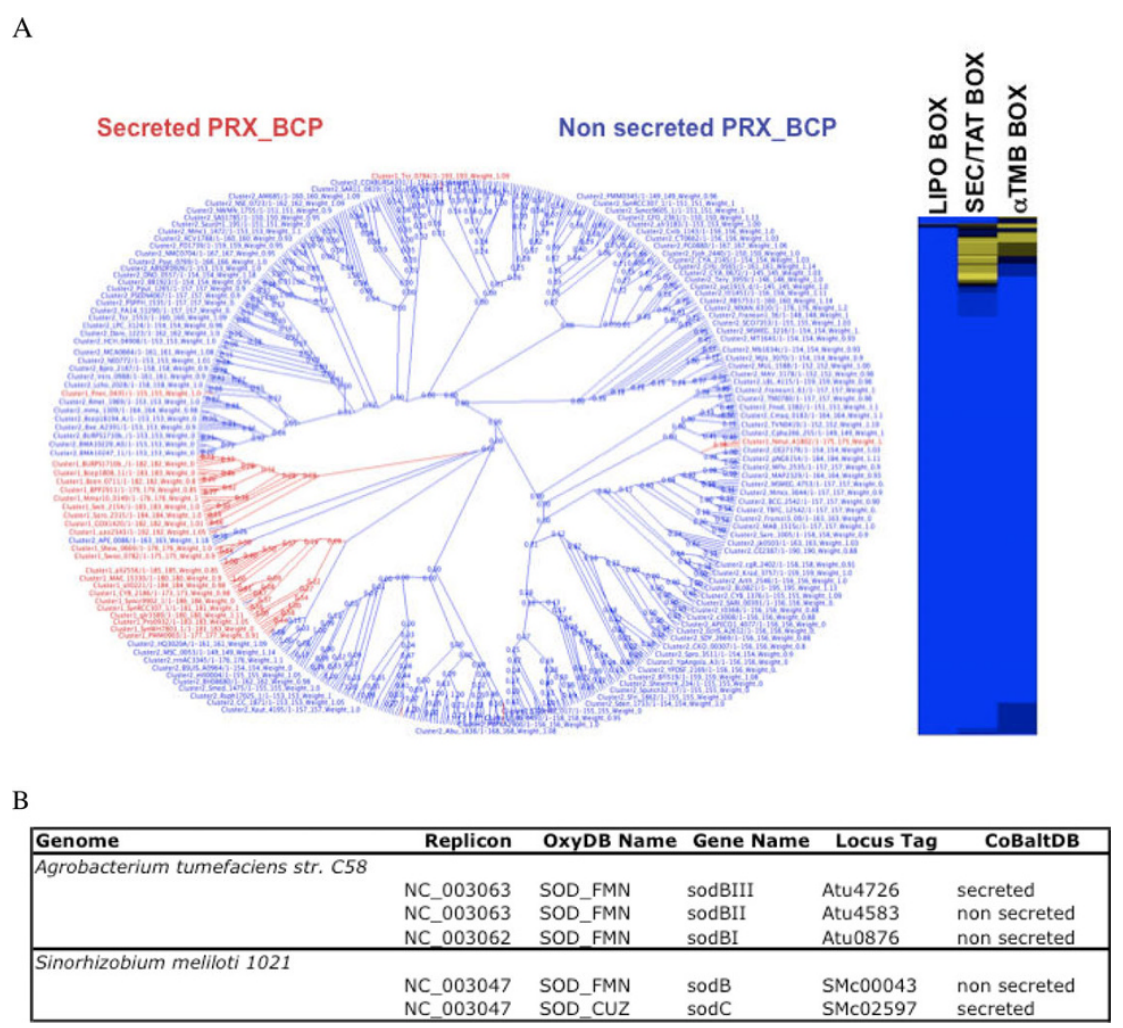

Figure 9 Using CoBalt for the analysis of orthologous and paralogous proteins. A: Phylogenetic tree of 1-Cystein peroxiredoxin PRX_BCP proteins and heat map of scores in each box for each PRX_BCP protein. B: OxyGene and CoBalt predictions for SOD in Agrobacterium tumefacins str. C58 and Sinorhizobium meliloti 1021.

tend to detect the presence of N-terminal signal peptides and predict cleavage sites. However the absence of an $\mathrm{N}$-terminal signal peptide does not systematically indicate that the protein is not secreted. Some proteins that are translocated via the Sec system might not necessarily exhibit an N-terminal signal peptide, such as the SodA protein of M. tuberculosis, which is dependent on SecA2 for secretion and lacks a classical signal sequence for protein export [103]. Furthermore, there is no systematic cleavage of the N-terminal signal peptide as it can serve as a cytoplasmic membrane anchor $[104,105]$. Another example: although type II and type V secretion systems generally require the presence of an $\mathrm{N}$-terminal signal peptide in order to utilise the sec pathway for translocation from cytoplasm to periplasm, type I and type III (and usually also type IV) systems can secrete a protein without any such signal $[28,106]$. Other proteins, such as Yop proteins exported by the Yersinia TTS system, have no classical sec-dependent signal sequences; however the information required to direct these proteins into the TTS pathway is contained within the $\mathrm{N}$-terminal coding region of each gene [107-109].

Some challenges still need to be addressed in the prediction of the subcellular localization of proteins. For instance, bioinformatics has recently focussed on predicting proteins secreted via other pathways $[110,111]$.

\section{Conclusion}

We have developed CoBaltDB, the first friendly interfaced database that compiles a large number of in silico subcellular predictions concerning whole bacterial and archaeal proteomes. Currently, CoBaltDB allows fast access to precomputed localizations for 2,548,292 proteins in 784 proteomes. It allows combined management of the predictions of 75 feature tools and 24 global tools and databases. New specialised prediction tools, algorithms and methods are continuously released, so CoBaltDB was designed to have the flexibility to facilitate inclusion of new tools or databases as required.

In general, our analysis indicates that both feature-based and general localization tools and databases have perform diversely in terms of specificity and sensitivity; the diversity arises mainly from the different sets of proteins used during the training process and from the limitations of the mathematical and statistical methodologies applied. In all our analyses with CoBaltDB, it became clear that that the combination and comparative analysis of results of heterogeneous tools improved the computational predictions, 
and contributed to identifying the limitations of each tool. Therefore, CoBaltDB can serve as a reference resource to facilitate interpretation of results and to provide a benchmark for accurate and effective in silico predictions of the subcellular localization of proteins. We hope that it will make a significant contribution to the exploitation of in silico subcellular localization predictions as users can easily create small datasets and determine their own thresholds for each predicted feature (type I or II SPs for example) or proteome. This is very important, as constructing an exhaustive "experimentally validated protein location" dataset is a time-consuming process -including identifying and reading all relevant papers- and as experimental findings about some subcellular locations are very limited.

\section{Availability and requirements}

\section{Database name: CoBaltDB}

Project home page: http://www.umr6026.univrennes1.fr/english/home/research/basic/software/cobalten

Operating system(s): Platform independent

Programming languages: Java, Python and BioPython

CoBaltDB package, requirements and documentations are freely available at http://www.umr6026.univ-rennes1. fr/english/home/research/basic/software/cobalten

Additional file 1: List of precomputed genomes (Excel). A table of all complete procaryotic genomes and corresponding replicons available in CoBaltDB.

Click here for file

[http://www.biomedcentral.com/content/supplementary/1471-2180-1088-S1.XLS ]

Additional file 2: Procaryotic subcellular localisation tools (HTML). This page is an inventory of all tools considered during the construction of CoBaltDB. The tools and databases related to the protein localization in procaryotic genomes are sorted by type of prediction. For each tool, a short description and the corresponding web link are displayed.

Click here for file

[http://www.biomedcentral.com/content/supplementary/1471-2180-1088-S2.PDF ]

Additional file 3: Monoderm and Diderm classification of genomes (PNG). Picture showing the cellular organization type (monoderm or diderm) for phylum in CoBaltDB.

Click here for file

[http://www.biomedcentral.com/content/supplementary/1471-2180-1088-S3.PNG ]

Additional file 4: Using CoBalt in comparative proteomics (PDF) Example of the lipoproteomes of E. coli K12 substrains, experimentally confirmed by EcoGene. Table1A: Prediction results for the 89 confirmed lipoproteins in the three substrains DH10B, MG1655 et W3110. Table1B: The lipoproteins that are not recognized by DOLOP have a sequence which does not match the DOLOP lipoBox pattern [LVI] [ASTVI] [ASG] [C] Click here for file

[http://www.biomedcentral.com/content/supplementary/1471-2180-1088-S4.PDF ]

\section{Abbreviations}

(AA): Amino acid; (aTMB): alpha-transmembrane; (CU): chaperone-usher; (ENP): extracellular nucleation-precipitation; (EXP): experimentally valitaded;
(FEA): flagella export apparatus; (FPE): fimbrilin-protein exporter; (GUI): Graphical User Interface; (HMM): Hidden Markov Model; (LIPO): lipoprotein; (NN): Neural Network; (PRX_BCPs): bacterioferritin comigratory protein homologs; (PSSM): Position Specific Scoring Matrix; (RE): regular expression; (ROS-RNS): Reactive Oxygen-Nitrogen species; (Sec): Sec apparatus; (SOAP): Simple Object Access Protocol; (SOD_CUZ): one copper-zinc superoxide dismutase; (SOD_FMN): iron-manganese superoxide dismutase; (SP): signal peptide; (SVM): support vector machine; (T1-7SS): Type (1-7) Secretion System; (Tat): Twin-arginine translocation; (TM): transmembrane; (Wss): WXG100 secretion system.

\section{Acknowledgements}

DG is supported by the Ministère de la Recherche. We wish to thank the bioinformatics platform of Biogenouest of Rennes for providing the hosting infrastructure.

\section{Authors' contributions}

DG designed and implemented the CoBaltDB database and the precomputing pipeline for automated data retrieval. SA and DG developed the user interface. CLM and FBH tested the database for functionality, and performed bioinformatics analyses leading to valuable suggestions on utility and design. CLM and SA helped coordinate the study. FBH conceived and managed the project. All authors participated in CoBaltDB design, contributed to workflow and interface designs and helped write the manuscript. All authors read and approved the final manuscript.

Received: 7 December 2009 Accepted: 23 March 2010

Published: 23 March 2010

\section{References}

1. Rost B, Liu J, Nair R, Wrzeszczynski KO, Ofran Y: Automatic prediction of protein function. Cell Mol Life Sci 2003, 60(12):2637-2650.

2. Nagy A, Hegyi H, Farkas K, Tordai H, Kozma E, Banyai L, Patthy L: Identification and correction of abnormal, incomplete and mispredicted proteins in public databases. BMC bioinformatics 2008, 9:353.

3. Desvaux M, Hebraud M, Talon R, Henderson IR: Secretion and subcellular localizations of bacterial proteins: a semantic awareness issue. Trends in microbiology 2009, 17(4):139-145.

4. De-la-Pena C, Lei Z, Watson BS, Sumner LW, Vivanco JM: Root-microbe communication through protein secretion. The Journal of biological chemistry 2008, 283(37):25247-25255.

5. Steward O, Pollack A, Rao A: Evidence that protein constituents of postsynaptic membrane specializations are locally synthesized: time course of appearance of recently synthesized proteins in synaptic junctions. Journal of neuroscience research 1991, 30(4):649-660.

6. Russo DM, Williams A, Edwards A, Posadas DM, Finnie C, Dankert M, Downie JA, Zorreguieta A: Proteins exported via the PrsD-PrsE type I secretion system and the acidic exopolysaccharide are involved in biofilm formation by Rhizobium leguminosarum. Journal of bacteriology 2006, 188(12):4474-4486.

7. Zhang L, Zhu Z, Jing $H$, Zhang J, Xiong $Y$, Yan M, Gao S, Wu LF, Xu J, Kan B: Pleiotropic effects of the twin-arginine translocation system on biofilm formation, colonization, and virulence in Vibrio cholerae. BMC microbiology 2009, 9:114.

8. De Buck E, Anne J, Lammertyn E: The role of protein secretion systems in the virulence of the intracellular pathogen Legionella pneumophila. Microbiology (Reading, England) 2007, 153(Pt 12):3948-3953.

9. Poueymiro M, Genin S: Secreted proteins from Ralstonia solanacearum: a hundred tricks to kill a plant. Current opinion in microbiology 2009, 12(1):44-52.

10. Shrivastava R, Miller JF: Virulence factor secretion and translocation by Bordetella species. Current opinion in microbiology 2009, 12(1):88-93.

11. Natale P, Bruser T, Driessen AJ: Sec- and Tat-mediated protein secretion across the bacterial cytoplasmic membrane-distinct translocases and mechanisms. Biochimica et biophysica acta 2008, 1778(9):1735-1756.

12. Papanikou E, Karamanou S, Economou A: Bacterial protein secretion through the translocase nanomachine. Nature reviews 2007, 5(11):839-851.

13. Muller M: Twin-arginine-specific protein export in Escherichia coli. Research in microbiology 2005, 156(2):131-136.

14. Lee PA, Tullman-Ercek D, Georgiou G: The bacterial twin-arginine translocation pathway. Annual review of microbiology 2006, 60:373-395. 
15. Albers SV, Szabo Z, Driessen AJ: Protein secretion in the Archaea: multiple paths towards a unique cell surface. Nature reviews 2006, 4(7):537-547.

16. Desvaux M, Parham NJ, Scott-Tucker A, Henderson IR: The general secretory pathway: a general misnomer? Trends in microbiology 2004, 12(7):306-309.

17. Delepelaire P: Type I secretion in gram-negative bacteria. Biochimica et biophysica acta 2004, 1694(1-3):149-161.

18. Holland IB, Schmitt L, Young J: Type 1 protein secretion in bacteria, the ABC-transporter dependent pathway (review). Molecular membrane biology 2005, 22(1-2):29-39.

19. Galan JE, Wolf-Watz H: Protein delivery into eukaryotic cells by type III secretion machines. Nature 2006, 444(7119):567-573

20. Ghosh P: Process of protein transport by the type III secretion system. Microbiol Mol Biol Rev 2004, 68(4):771-795.

21. Medini D, Covacci A, Donati C: Protein homology network families reveal step-wise diversification of Type III and Type IV secretion systems. PLoS computational biology 2006, 2(12):e173.

22. Pukatzki S, McAuley SB, Miyata ST: The type VI secretion system: translocation of effectors and effector-domains. Current opinion in microbiology 2009, 12(1):11-17.

23. Filloux A, Hachani A, Bleves S: The bacterial type VI secretion machine: yet another player for protein transport across membranes. Microbiology (Reading, England) 2008, 154(Pt 6):1570-1583.

24. Desvaux M, Hebraud M, Henderson IR, Pallen MJ: Type III secretion: what's in a name? Trends in microbiology 2006, 14(4):157-160.

25. Coulthurst SJ, Palmer T: A new way out: protein localization on the bacterial cell surface via Tat and a novel Type II secretion system. Molecular microbiology 2008, 69(6):1331-1335.

26. Cianciotto NP: Type II secretion: a protein secretion system for all seasons. Trends in microbiology 2005, 13(12):581-588.

27. Mueller CA, Broz P, Cornelis GR: The type III secretion system tip complex and translocon. Molecular microbiology 2008, 68(5):1085-1095.

28. Henderson IR, Navarro-Garcia F, Desvaux M, Fernandez RC, Ala'Aldeen D: Type V protein secretion pathway: the autotransporter story. Microbiol Mol Biol Rev 2004, 68(4):692-744.

29. Desvaux M, Parham NJ, Henderson IR: Type V protein secretion: simplicity gone awry? Current issues in molecular biology 2004, 6(2):111-124.

30. Nuccio SP, Baumler AJ: Evolution of the chaperone/usher assembly pathway: fimbrial classification goes Greek. Microbiol Mol Biol Rev 2007, 71(4):551-575

31. Sauer FG, Remaut H, Hultgren SJ, Waksman G: Fiber assembly by the chaperone-usher pathway. Biochimica et biophysica acta 2004, 1694(13):259-267.

32. Kostakioti M, Newman CL, Thanassi DG, Stathopoulos C: Mechanisms of protein export across the bacterial outer membrane. Journal of bacteriology 2005, 187(13):4306-4314.

33. Bitter W, Houben EN, Luirink J, Appelmelk BJ: Type VII secretion in mycobacteria: classification in line with cell envelope structure. Trends in microbiology 2009, 17(8):337-338.

34. Desvaux M, Khan A, Scott-Tucker A, Chaudhuri RR, Pallen MJ, Henderson IR: Genomic analysis of the protein secretion systems in Clostridium acetobutylicum ATCC 824. Biochimica et biophysica acta 2005, 1745(2):223-253.

35. Peabody CR, Chung YJ, Yen MR, Vidal-Ingigliardi D, Pugsley AP, Saier MH Jr: Type II protein secretion and its relationship to bacterial type IV pili and archaeal flagella. Microbiology (Reading, England) 2003, 149(Pt 11):3051-3072

36. Aldridge $P$, Hughes $K T$ : How and when are substrates selected for type III secretion? Trends in microbiology 2001, 9(5):209-214.

37. Pallen MJ: The ESAT-6/WXG100 superfamily - and a new Gram-positive secretion system? Trends in microbiology 2002, 10(5):209-212.

38. Desvaux M, Hebraud M, Talon R, Henderson IR: Outer membrane translocation: numerical protein secretion nomenclature in question in mycobacteria. Trends in microbiology 2009, 17(8):338-340.

39. von Heijne G: Patterns of amino acids near signal-sequence cleavage sites. European journal of biochemistry/FEBS 1983, 133(1):17-21.

40. von Heijne G: A new method for predicting signal sequence cleavage sites. Nucleic acids research 1986, 14(11):4683-4690.

41. McGeoch DJ: On the predictive recognition of signal peptide sequences. Virus research 1985, 3(3):271-286.
42. Ladunga I, Czako F, Csabai I, Geszti T: Improving signal peptide prediction accuracy by simulated neural network. Comput Appl Biosci 1991, 7(4):485-487.

43. Schneider $G$, Rohlk S, Wrede P: Analysis of cleavage-site patterns in protein precursor sequences with a perceptron-type neural network. Biochemical and biophysical research communications 1993, 194(2):951-959.

44. Plewczynski D, Slabinski L, Ginalski K, Rychlewski L: Prediction of signal peptides in protein sequences by neural networks. Acta biochimica Polonica 2008, 55(2):261-267.

45. Nielsen $H$, Krogh A: Prediction of signal peptides and signal anchors by a hidden Markov model. Proceedings/International Conference on Intelligent Systems for Molecular Biology; ISMB 1998, 6:122-130.

46. Bendtsen JD, Nielsen $H$, von Heijne $G$, Brunak S: Improved prediction of signal peptides: SignalP 3.0. Journal of molecular biology 2004, 340(4):783-795.

47. Nielsen $\mathrm{H}$, Engelbrecht J, Brunak S, von Heijne G: Identification of prokaryotic and eukaryotic signal peptides and prediction of their cleavage sites. Protein Eng 1997, 10(1):1-6.

48. Kall L, Krogh A, Sonnhammer EL: A combined transmembrane topology and signal peptide prediction method. $J$ Mol Biol 2004, 338(5):1027-1036.

49. Kall L, Krogh A, Sonnhammer EL: Advantages of combined transmembrane topology and signal peptide prediction-the Phobius web server. Nucleic Acids Res 2007, 35 Web Server: W429-432.

50. Zhang Z, Henzel WJ: Signal peptide prediction based on analysis of experimentally verified cleavage sites. Protein Sci 2004, 13(10):2819-2824

51. Berks BC: A common export pathway for proteins binding complex redox cofactors? Molecular microbiology 1996, 22(3):393-404.

52. Rose RW, Bruser T, Kissinger JC, Pohlschroder M: Adaptation of protein secretion to extremely high-salt conditions by extensive use of the twinarginine translocation pathway. Molecular microbiology 2002, 45(4):943-950.

53. Bendtsen JD, Nielsen $H$, Widdick D, Palmer T, Brunak S: Prediction of twinarginine signal peptides. BMC Bioinformatics 2005, 6:167

54. von Heijne $\mathrm{G}$ : The structure of signal peptides from bacterial lipoproteins. Protein engineering 1989, 2(7):531-534

55. Sankaran K, Gan K, Rash B, Qi HY, Wu HC, Rick PD: Roles of histidine-103 and tyrosine-235 in the function of the prolipoprotein diacylglyceryl transferase of Escherichia coli. Journal of bacteriology 1997, 179(9):2944-2948.

56. Berven FS, Karlsen OA, Straume AH, Flikka K, Murrell JC, Fjellbirkeland A, Lillehaug JR, Eidhammer I, Jensen HB: Analysing the outer membrane subproteome of Methylococcus capsulatus (Bath) using proteomics and novel biocomputing tools. Archives of microbiology 2006, 184(6):362-377.

57. Babu MM, Priya ML, Selvan AT, Madera M, Gough J, Aravind L, Sankaran K A database of bacterial lipoproteins (DOLOP) with functional assignments to predicted lipoproteins. Journal of bacteriology 2006, 188(8):2761-2773.

58. Bagos PG, Tsirigos KD, Liakopoulos TD, Hamodrakas SJ: Prediction of lipoprotein signal peptides in Gram-positive bacteria with a Hidden Markov Model. J Proteome Res 2008, 7(12):5082-5093.

59. Juncker AS, Willenbrock H, Von Heijne G, Brunak S, Nielsen H, Krogh A: Prediction of lipoprotein signal peptides in Gram-negative bacteria. Protein Sci 2003, 12(8):1652-1662.

60. Klein $\mathrm{P}$, Kanehisa M, DeLisi C: The detection and classification of membrane-spanning proteins. Biochimica et biophysica acta 1985, 815(3):468-476.

61. Claros MG, von Heijne G: TopPred II: an improved software for membrane protein structure predictions. Comput Appl Biosci 1994 10(6):685-686

62. Hirokawa T, Boon-Chieng S, Mitaku S: SOSUI: classification and secondary structure prediction system for membrane proteins. Bioinformatics (Oxford, England) 1998, 14(4):378-379.

63. Jayasinghe S, Hristova K, White SH: Energetics, stability, and prediction of transmembrane helices. Journal of molecular biology 2001, 312(5):927-934.

64. Ganapathiraju M, Jursa CJ, Karimi HA, Klein-Seetharaman J: TMpro web server and web service: transmembrane helix prediction through amino acid property analysis. Bioinformatics 2007, 23(20):2795-2796.

65. Deber CM, Wang C, Liu LP, Prior AS, Agrawal S, Muskat BL, Cuticchia AJ: TM Finder: a prediction program for transmembrane protein segments using a combination of hydrophobicity and nonpolar phase helicity scales. Protein Sci 2001, 10(1):212-219. 
66. Jones DT, Taylor WR, Thornton JM: A model recognition approach to the prediction of all-helical membrane protein structure and topology. Biochemistry 1994, 33(10):3038-3049.

67. Persson B, Argos P: Prediction of membrane protein topology utilizing multiple sequence alignments. Journal of protein chemistry 1997, 16(5):453-457.

68. Rost B, Fariselli P, Casadio R: Topology prediction for helical transmembrane proteins at $86 \%$ accuracy. Protein Sci 1996, 5(8):1704-1718.

69. Aloy P, Cedano J, Oliva B, Aviles FX, Querol E: 'TransMem': a neural network implemented in Excel spreadsheets for predicting transmembrane domains of proteins. Comput Appl Biosci 1997, 13(3):231-234.

70. Krogh A, Larsson B, von Heijne G, Sonnhammer EL: Predicting transmembrane protein topology with a hidden Markov model: application to complete genomes. Journal of molecular biology 2001, 305(3):567-580.

71. Tusnady GE, Simon I: The HMMTOP transmembrane topology prediction server. Bioinformatics 2001, 17(9):849-850.

72. Viklund $\mathrm{H}$, Elofsson $\mathrm{A}$ : Best alpha-helical transmembrane protein topology predictions are achieved using hidden Markov models and evolutionary information. Protein Sci 2004, 13(7):1908-1917.

73. Yuan Z, Mattick JS, Teasdale RD: SVMtm: support vector machines to predict transmembrane segments. Journal of computational chemistry 2004, 25(5):632-636.

74. Garrow AG, Agnew A, Westhead DR: TMB-Hunt: an amino acid composition based method to screen proteomes for beta-barrel transmembrane proteins. BMC bioinformatics 2005, 6:56.

75. Garrow AG, Westhead DR: A consensus algorithm to screen genomes for novel families of transmembrane beta barrel proteins. Proteins 2007, 69(1):8-18.

76. Bagos PG, Liakopoulos TD, Hamodrakas SJ: Evaluation of methods for predicting the topology of beta-barrel outer membrane proteins and a consensus prediction method. BMC bioinformatics 2005, 6:7.

77. Martelli PL, Fariselli P, Krogh A, Casadio R: A sequence-profile-based HMM for predicting and discriminating beta barrel membrane proteins. Bioinformatics (Oxford, England) 2002, 18(Suppl 1):S46-53.

78. Bigelow HR, Petrey DS, Liu J, Przybylski D, Rost B: Predicting transmembrane beta-barrels in proteomes. Nucleic acids research 2004 32(8):2566-2577

79. Randall A, Cheng J, Sweredoski M, Baldi P: TMBpro: secondary structure, beta-contact and tertiary structure prediction of transmembrane betabarrel proteins. Bioinformatics (Oxford, England) 2008, 24(4):513-520.

80. Bigelow $\mathrm{H}$, Rost B: PROFtmb: a web server for predicting bacterial transmembrane beta barrel proteins. Nucleic acids research 2006, , 34 Web Server: W186-188.

81. Hu J, Yan C: A method for discovering transmembrane beta-barrel proteins in Gram-negative bacterial proteomes. Computational biology and chemistry 2008, 32(4):298-301.

82. Waldispuhl J, Berger B, Clote P, Steyaert JM: transFold: a web server for predicting the structure and residue contacts of transmembrane betabarrels. Nucleic acids research 2006, , 34 Web Server: W189-193.

83. Zhai $Y$, Saier MH Jr: The beta-barrel finder (BBF) program, allowing identification of outer membrane beta-barrel proteins encoded within prokaryotic genomes. Protein Sci 2002, 11(9):2196-2207.

84. Berven FS, Flikka K, Jensen HB, Eidhammer I: BOMP: a program to predict integral beta-barrel outer membrane proteins encoded within genomes of Gram-negative bacteria. Nucleic Acids Res 2004, , 32 Web Server: W394-399.

85. Bagos PG, Liakopoulos TD, Spyropoulos IC, Hamodrakas SJ: PRED-TMBB: a web server for predicting the topology of beta-barrel outer membrane proteins. Nucleic Acids Res 2004, 32 Web Server: W400-404.

86. Park KJ, Gromiha MM, Horton P, Suwa M: Discrimination of outer membrane proteins using support vector machines. Bioinformatics 2005 21(23):4223-4229.

87. Ou YY, Gromiha MM, Chen SA, Suwa M: TMBETADISC-RBF: Discrimination of beta-barrel membrane proteins using RBF networks and PSSM profiles. Computational biology and chemistry 2008, 32(3):227-231.

88. Billion A, Ghai R, Chakraborty T, Hain T: Augur-a computational pipeline for whole genome microbial surface protein prediction and classification. Bioinformatics 2006, 22(22):2819-2820.
89. Zhou M, Boekhorst J, Francke C, Siezen RJ: LocateP: genome-scale subcellular-location predictor for bacterial proteins. BMC bioinformatics 2008, 9:173.

90. Choo KH, Tan TW, Ranganathan S: SPdb-a signal peptide database. BMC bioinformatics 2005, 6:249.

91. Rey S, Acab M, Gardy JL, Laird MR, deFays K, Lambert C, Brinkman FS: PSORTdb: a protein subcellular localization database for bacteria. Nucleic Acids Res 2005, , 33 Database: D164-168.

92. Park S, Yang JS, Jang SK, Kim S: Construction of Functional Interaction Networks through Consensus Localization Predictions of the Human Proteome. J Proteome Res 2009, 8(7):3367-3376.

93. Restrepo-Montoya D, Vizcaino C, Nino LF, Ocampo M, Patarroyo ME, Patarroyo MA: Validating subcellular localization prediction tools with mycobacterial proteins. BMC Bioinformatics 2009, 10:134.

94. Shen $Y Q$, Burger $G$ : 'Unite and conquer': enhanced prediction of protein subcellular localization by integrating multiple specialized tools. $B M C$ Bioinformatics 2007, 8:420.

95. Gupta RS: The natural evolutionary relationships among prokaryotes. Critical reviews in microbiology 2000, 26(2):111-131.

96. Rachel R, Wyschkony I, Riehl S, Huber H: The ultrastructure of Ignicoccus: evidence for a novel outer membrane and for intracellular vesicle budding in an archaeon. Archaea (Vancouver, BC) 2002, 1(1):9-18.

97. Rudd KE: EcoGene: a genome sequence database for Escherichia coli K12. Nucleic Acids Res 2000, 28(1):60-64.

98. Itoh T, Okayama T, Hashimoto H, Takeda J, Davis RW, Mori H, Gojobori T: A low rate of nucleotide changes in Escherichia coli K-12 estimated from a comparison of the genome sequences between two different substrains. FEBS letters 1999, 450(1-2):72-76.

99. Durfee T, Nelson R, Baldwin S, Plunkett G, Burland V, Mau B, Petrosino JF, Qin X, Muzny DM, Ayele $M$, et al: The complete genome sequence of Escherichia coli $\mathrm{DH} 10 \mathrm{~B}$ : insights into the biology of a laboratory workhorse. J Bacteriol 2008, 190(7):2597-2606.

100. Peterson KM, Mekalanos JJ: Characterization of the Vibrio cholerae ToxR regulon: identification of novel genes involved in intestinal colonization. Infection and immunity 1988, 56(11):2822-2829.

101. Miyadai H, Tanaka-Masuda K, Matsuyama S, Tokuda H: Effects of lipoprotein overproduction on the induction of DegP $(\mathrm{HtrA})$ involved in quality control in the Escherichia coli periplasm. The Journal of biological chemistry 2004, 279(38):39807-39813.

102. Thybert D, Avner S, Lucchetti-Miganeh C, Cheron A, Barloy-Hubler F: OxyGene: an innovative platform for investigating oxidative-response genes in whole prokaryotic genomes. BMC genomics 2008, 9:637.

103. Braunstein M, Espinosa BJ, Chan J, Belisle JT, Jacobs WR Jr: SecA2 functions in the secretion of superoxide dismutase $A$ and in the virulence of Mycobacterium tuberculosis. Molecular microbiology 2003, 48(2):453-464.

104. Goder V, Spiess M: Topogenesis of membrane proteins: determinants and dynamics. FEBS letters 2001, 504(3):87-93.

105. Martoglio B, Dobberstein B: Signal sequences: more than just greasy peptides. Trends in cell biology 1998, 8(10):410-415.

106. Bingle LE, Bailey CM, Pallen MJ: Type VI secretion: a beginner's guide. Current opinion in microbiology 2008, 11(1):3-8.

107. Anderson DM, Schneewind O: A mRNA signal for the type III secretion of Yop proteins by Yersinia enterocolitica. Science (New York, NY) 1997, 278(5340):1140-1143.

108. Anderson DM, Schneewind O: Yersinia enterocolitica type III secretion: an mRNA signal that couples translation and secretion of YopQ. Molecular microbiology 1999, 31(4):1139-1148.

109. Michiels T, Wattiau P, Brasseur R, Ruysschaert JM, Cornelis G: Secretion of Yop proteins by Yersiniae. Infection and immunity 1990, 58(9):2840-2849.

110. Lower M, Schneider G: Prediction of type III secretion signals in genomes of gram-negative bacteria. PLoS One 2009, 4(6):e5917.

111. Arnold R, Brandmaier S, Kleine F, Tischler P, Heinz E, Behrens S, Niinikoski A, Mewes HW, Horn M, Rattei T: Sequence-based prediction of type III secreted proteins. PLoS pathogens 2009, 5(4):e1000376.

112. Hiller K, Grote A, Scheer M, Munch R, Jahn D: PrediSi: prediction of signal peptides and their cleavage positions. Nucleic Acids Res 2004, , 32 Web Server: W375-379.

113. Gomi M, Sonoyama M, Mitaku S: High performance system for signal peptide prediction: SOSUlsignal. Chem-Bio Informatics Journal 2004, 4(4):142-147. 
114. Mitaku S, Hirokawa T, Tsuji T: Amphiphilicity index of polar amino acids as an aid in the characterization of amino acid preference at membranewater interfaces. Bioinformatics 2002, 18(4):608-616.

115. Juretic D, Zoranic L, Zucic D: Basic charge clusters and predictions of membrane protein topology. J Chem Inf Comput Sci 2002, 42(3):620-632.

116. Bagos PG, Liakopoulos TD, Hamodrakas SJ: Finding beta-barrel outer membrane proteins with a Markov Chain Model. WSEAS Transactions on Biology and Biomedecine 2004, 1(2):186-189.

117. Gromiha MM, Ahmad S, Suwa M: TMBETA-NET: discrimination and prediction of membrane spanning beta-strands in outer membrane proteins. Nucleic Acids Res 2005, , 33 Web Server: W164-167.

118. Garrow AG, Agnew A, Westhead DR: TMB-Hunt: a web server to screen sequence sets for transmembrane beta-barrel proteins. Nucleic Acids Res 2005, , 33 Web Server: W188-192.

119. Lu Z, Szafron D, Greiner R, Lu P, Wishart DS, Poulin B, Anvik J, Macdonell C, Eisner R: Predicting subcellular localization of proteins using machinelearned classifiers. Bioinformatics 2004, 20(4):547-556.

120. Matsuda S, Vert JP, Saigo H, Ueda N, Toh H, Akutsu T: A novel representation of protein sequences for prediction of subcellular location using support vector machines. Protein Sci 2005, 14(11):2804-2813.

121. Hua S, Sun Z: Support vector machine approach for protein subcellular localization prediction. Bioinformatics 2001, 17(8):721-728.

122. Niu B, Jin YH, Feng KY, Lu WC, Cai YD, Li GZ: Using AdaBoost for the prediction of subcellular location of prokaryotic and eukaryotic proteins. Molecular diversity 2008, 12(1):41-45.

123. Imai K, Asakawa N, Tsuji T, Akazawa F, Ino A, Sonoyama M, Mitaku S: SOSUIGramN: high performance prediction for sub-cellular localization of proteins in Gram-negative bacteria. Bioinformation 2008, 2(9):417-421.

124. Horler RS, Butcher A, Papangelopoulos N, Ashton PD, Thomas GH: EchoLOCATION: an in silico analysis of the subcellular locations of Escherichia coli proteins and comparison with experimentally derived locations. Bioinformatics 2009, 25(2):163-166.

125. Fernando SA, Selvarani P, Das S, Kumar Ch K, Mondal S, Ramakumar S, Sekar K: THGS: a web-based database of Transmembrane Helices in Genome Sequences. Nucleic Acids Res 2004, , 32 Database: D125-128.

126. Litou ZI, Bagos PG, Tsirigos KD, Liakopoulos TD, Hamodrakas SJ: Prediction of cell wall sorting signals in gram-positive bacteria with a hidden markov model: application to complete genomes. Journal of bioinformatics and computational biology 2008, 6(2):387-401

127. Remmert M, Linke D, Lupas AN, Soding J: HHomp-prediction and classification of outer membrane proteins. Nucleic Acids Res 2009, , 37 Web Server: W446-451.

128. Saleh MT, Fillon M, Brennan PJ, Belisle JT: Identification of putative exported/secreted proteins in prokaryotic proteomes. Gene 2001, 269(12):195-204.

129. Bagos PG, Tsirigos KD, Plessas SK, Liakopoulos TD, Hamodrakas SJ: Prediction of signal peptides in archaea. Protein Eng Des Sel 2009, 22(1):27-35.

130. Ikeda M, Arai M, Okuno T, Shimizu T: TMPDB: a database of experimentally-characterized transmembrane topologies. Nucleic Acids Res 2003, 31(1):406-409.

131. Tusnady GE, Kalmar L, Simon I: TOPDB: topology data bank of transmembrane proteins. Nucleic Acids Res 2008, , 36 Database: D234-239.

132. Menne KM, Hermjakob $H$, Apweiler R: A comparison of signal sequence prediction methods using a test set of signal peptides. Bioinformatics 2000, 16(8):741-742.

133. Taylor PD, Toseland CP, Attwood TK, Flower DR: LIPPRED: A web server for accurate prediction of lipoprotein signal sequences and cleavage sites. Bioinformation 2006, 1(5):176-179.

134. Fariselli P, Finocchiaro G, Casadio R: SPEPlip: the detection of signal peptide and lipoprotein cleavage sites. Bioinformatics 2003, 19(18):2498-2499.

135. Bendtsen JD, Kiemer L, Fausboll A, Brunak S: Non-classical protein secretion in bacteria. BMC Microbiol 2005, 5:58.

136. Shen HB, Chou KC: Signal-3L: A 3-layer approach for predicting signal peptides. Biochem Biophys Res Commun 2007, 363(2):297-303.

137. Chou KC, Shen HB: Signal-CF: a subsite-coupled and window-fusing approach for predicting signal peptides. Biochem Biophys Res Commun 2007, 357(3):633-640
138. Frank K, Sippl MJ: High-performance signal peptide prediction based on sequence alignment techniques. Bioinformatics 2008, 24(19):2172-2176.

139. Szabo Z, Stahl AO, Albers SV, Kissinger JC, Driessen AJ, Pohlschroder M: Identification of diverse archaeal proteins with class III signal peptides cleaved by distinct archaeal prepilin peptidases. J Bacteriol 2007, 189(3):772-778.

140. Hiss JA, Resch E, Schreiner A, Meissner M, Starzinski-Powitz A, Schneider G: Domain organization of long signal peptides of single-pass integral membrane proteins reveals multiple functional capacity. PLoS One 2008 3(7):e2767.

141. Reynolds SM, Kall L, Riffle ME, Bilmes JA, Noble WS: Transmembrane topology and signal peptide prediction using dynamic bayesian networks. PLoS Comput Biol 2008, 4(11):e1000213.

142. Viklund $H$, Elofsson A: OCTOPUS: improving topology prediction by twotrack ANN-based preference scores and an extended topological grammar. Bioinformatics 2008, 24(15):1662-1668.

143. Viklund H, Bernsel A, Skwark M, Elofsson A: SPOCTOPUS: a combined predictor of signal peptides and membrane protein topology. Bioinformatics 2008, 24(24):2928-2929.

144. Shen H, Chou JJ: MemBrain: improving the accuracy of predicting transmembrane helices. PLoS One 2008, 3(6):e2399.

145. Cserzo M, Wallin E, Simon I, von Heijne G, Elofsson A: Prediction of transmembrane alpha-helices in prokaryotic membrane proteins: the dense alignment surface method. Protein Eng 1997, 10(6):673-676.

146. Bagos PG, Liakopoulos TD, Hamodrakas SJ: Algorithms for incorporating prior topological information in HMMs: application to transmembrane proteins. BMC Bioinformatics 2006, 7:189.

147. Lo A, Chiu HS, Sung TY, Lyu PC, Hsu WL: Enhanced membrane protein topology prediction using a hierarchical classification method and a new scoring function. J Proteome Res 2008, 7(2):487-496.

148. Zhou H, Zhou Y: Predicting the topology of transmembrane helical proteins using mean burial propensity and a hidden-Markov-modelbased method. Protein Sci 2003, 12(7):1547-1555.

149. Pashou EE, Litou ZI, Liakopoulos TD, Hamodrakas SJ: waveTM: waveletbased transmembrane segment prediction. Silico Biol 2004, 4(2):127-131.

150. Pasquier C, Promponas VJ, Palaios GA, Hamodrakas JS, Hamodrakas SJ: A novel method for predicting transmembrane segments in proteins based on a statistical analysis of the SwissProt database: the PRED-TMR algorithm. Protein Eng 1999, 12(5):381-385.

151. Peris P, Lopez D, Campos M: IgTM: an algorithm to predict transmembrane domains and topology in proteins. BMC Bioinformatics 2008, 9:367.

152. Bernsel A, Viklund $H$, Hennerdal A, Elofsson A: TOPCONS: consensus prediction of membrane protein topology. Nucleic Acids Res 2009, , 37 Web Server: W465-468.

153. Zhou H, Zhang C, Liu S, Zhou Y: Web-based toolkits for topology prediction of transmembrane helical proteins, fold recognition, structure and binding scoring, folding-kinetics analysis and comparative analysis of domain combinations. Nucleic Acids Res 2005, 33 Web Server: W193-197.

154. Arai M, Mitsuke H, Ikeda M, Xia JX, Kikuchi T, Satake M, Shimizu T: ConPred II: a consensus prediction method for obtaining transmembrane topology models with high reliability. Nucleic Acids Res 2004, 32 Web Server: W390-393.

155. Jones DT: Improving the accuracy of transmembrane protein topology prediction using evolutionary information. Bioinformatics 2007, 23(5):538-544.

156. Adamczak R, Porollo A, Meller J: Combining prediction of secondary structure and solvent accessibility in proteins. Proteins 2005, 59(3):467-475.

157. Ganapathiraju M, Balakrishnan N, Reddy R, Klein-Seetharaman J: Transmembrane helix prediction using amino acid property features and latent semantic analysis. BMC Bioinformatics 2008, 9(Suppl 1):S4.

158. Jones DT: Protein secondary structure prediction based on positionspecific scoring matrices. J Mol Biol 1999, 292(2):195-202.

159. Bryson K, McGuffin LJ, Marsden RL, Ward JJ, Sodhi JS, Jones DT: Protein structure prediction servers at University College London. Nucleic Acids Res 2005, , 33 Web Server: W36-38.

160. Combet C, Blanchet C, Geourjon C, Deleage G: NPS@: network protein sequence analysis. Trends Biochem Sci 2000, 25(3):147-150. 
161. Karplus K: SAM-T08, HMM-based protein structure prediction. Nucleic Acids Res 2009, 37 Web Server: W492-497.

162. Pollastri G, McLysaght A: Porter: a new, accurate server for protein secondary structure prediction. Bioinformatics 2005, 21(8):1719-1720.

163. Kahsay RY, Gao G, Liao L: An improved hidden Markov model for transmembrane protein detection and topology prediction and its applications to complete genomes. Bioinformatics 2005, 21(9):1853-1858.

164. Lin K, Simossis VA, Taylor WR, Heringa J: A simple and fast secondary structure prediction method using hidden neural networks. Bioinformatics 2005, 21(2):152-159.

165. Chou KC, Shen HB: MemType-2L: a web server for predicting membrane proteins and their types by incorporating evolution information through Pse-PSSM. Biochem Biophys Res Commun 2007, 360(2):339-345.

166. Yu CS, Chen YC, Lu CH, Hwang JK: Prediction of protein subcellular localization. Proteins 2006, 64(3):643-651.

167. Su EC, Chiu HS, Lo A, Hwang JK, Sung TY, Hsu WL: Protein subcellular localization prediction based on compartment-specific features and structure conservation. BMC Bioinformatics 2007, 8:330

168. Bhasin M, Garg A, Raghava GP: PSLpred: prediction of subcellular localization of bacterial proteins. Bioinformatics 2005, 21(10):2522-2524

169. Chou KC, Shen HB: Large-scale predictions of gram-negative bacterial protein subcellular locations. J Proteome Res 2006, 5(12):3420-3428.

170. Shen HB, Chou KC: Gpos-PLoc: an ensemble classifier for predicting subcellular localization of Gram-positive bacterial proteins. Protein Eng Des Sel 2007, 20(1):39-46.

171. Nair R, Rost B: Mimicking cellular sorting improves prediction of subcellular localization. J Mol Biol 2005, 348(1):85-100.

172. Jia P, Qian Z, Zeng Z, Cai Y, Li Y: Prediction of subcellular protein localization based on functional domain composition. Biochem Biophys Res Commun 2007, 357(2):366-370.

173. Rashid M, Saha S, Raghava GP: Support Vector Machine-based method for predicting subcellular localization of mycobacterial proteins using evolutionary information and motifs. BMC Bioinformatics 2007, 8:337.

174. Setubal JC, Reis M, Matsunaga J, Haake DA: Lipoprotein computational prediction in spirochaetal genomes. Microbiology 2006, 152(Pt 1):113-121.

175. Montgomerie S, Cruz JA, Shrivastava S, Arndt D, Berjanskii M, Wishart DS: PROTEUS2: a web server for comprehensive protein structure prediction and structure-based annotation. Nucleic Acids Res 2008, 36 Web Server: W202-209.

176. Pasquier C, Hamodrakas SJ: An hierarchical artificial neural network system for the classification of transmembrane proteins. Protein Eng 1999, 12(8):631-634.

177. Taylor PD, Attwood TK, Flower DR: BPROMPT: A consensus server for membrane protein prediction. Nucleic Acids Res 2003, 31(13):3698-3700

178. Liakopoulos TD, Pasquier C, Hamodrakas SJ: A novel tool for the prediction of transmembrane protein topology based on a statistical analysis of the SwissProt database: the OrienTM algorithm. Protein Eng 2001, 14(6):387-390.

179. Raghava GP: APSSP2: A combination method for protein secondary structure prediction based on neural network and example based learning. CASP5 2002, A-132.

180. Simossis VA, Heringa J: PRALINE: a multiple sequence alignment toolbox that integrates homology-extended and secondary structure information. Nucleic Acids Res 2005, 33 Web Server: W289-294.

181. Lomize MA, Lomize AL, Pogozheva ID, Mosberg HI: OPM: orientations of proteins in membranes database. Bioinformatics 2006, 22(5):623-625.

182. Jayasinghe $\mathrm{S}$, Hristova K, White SH: MPtopo: A database of membrane protein topology. Protein Sci 2001, 10(2):455-458.

183. Tusnady GE, Dosztanyi Z, Simon I: PDB_TM: selection and membrane localization of transmembrane proteins in the protein data bank. Nucleic Acids Res 2005, 33 Database: D275-278.

184. Gromiha MM, Yabuki Y, Kundu S, Suharnan S, Suwa M: TMBETA-GENOME: database for annotated beta-barrel membrane proteins in genomic sequences. Nucleic Acids Res 2007, , 35 Database: D314-316.

185. Rost B, Yachdav G, Liu J: The PredictProtein server. Nucleic Acids Res 2004 32 Web Server: W321-326.

186. Yun H, Lee JW, Jeong J, Chung J, Park JM, Myoung HN, Lee SY: EcoProDB: the Escherichia coli protein database. Bioinformatics 2007, 23(18):2501-2503.
187. Nair R, Rost B: LOCnet and LOCtarget: sub-cellular localization for structural genomics targets. Nucleic Acids Res 2004, 32 Web Server: W517-521.

188. Zhang S, Xia X, Shen J, Zhou Y, Sun Z: DBMLoc: a Database of proteins with multiple subcellular localizations. BMC Bioinformatics 2008, 9:127.

doi:10.1186/1471-2180-10-88

Cite this article as: Goudenège et al: CoBaltDB: Complete bacterial and archaeal orfeomes subcellular localization database and associated resources. BMC Microbiology 2010 10:88.

\section{Submit your next manuscript to BioMed Central and take full advantage of:}

- Convenient online submission

- Thorough peer review

- No space constraints or color figure charges

- Immediate publication on acceptance

- Inclusion in PubMed, CAS, Scopus and Google Scholar

- Research which is freely available for redistribution

Submit your manuscript at www.biomedcentral.com/submit
C Biomed Central 